3. In der nachstehenden Arbeit von Bingel ${ }^{8}$ wird für die Lage des tiefsten angeregten Zustandes des Benzylradikals nach der Methode der Molekülbahnen ein Wert von $\sim 21300 \mathrm{~cm}^{-1}$ und nach dem Modell der freien Elektronen der Wert von $\sim 21800 \mathrm{~cm}^{-1}$ errechnet. Nach der von uns getroffenen Zuordnung entsprechen diese Zahlen dem experimentellen Wert $\sim 22330 \mathrm{~cm}^{-1}$.

Die Übereinstimmung ist sehr befriedigend. Vor allen Dingen weist sie darauf hin, daß die um mehr als $9000 \mathrm{~cm}^{-1}$ höher liegende Absorption von Porter nur einem Übergang nach einem höher angeregten Zustand entsprechen kann. Außerdem ergibt die Theorie, daß der Absorptionsübergang zum tiefsten angeregten Zustand des Benzylradi- kals eine relativ kleine Wahrscheinlichkeit hat. Sie zeigt auch, daß noch höher angeregte Zustände mit großer Übergangswahrscheinlichkeit existieren. Dem Übergang zu einem solchen Term muß die Portersche Absorption entsprechen. Da Porter einen solchen starken Übergang bisher nur relativ schwach nachweist, ist es plausibel, daß sich der erste schwache Übergang bei $22330 \mathrm{~cm}^{-1}$ der Beobachtung entzogen hat.

Die in dieser Arbeit angestellten Betrachtungen lassen sich auch ganz analog auf andere aromatische Substanzen ausdehnen.

${ }^{8}$ W. Bingel, Z. Naturforschg. 10a, 462 [1955].

\title{
Die Elektronenstruktur und Lichtabsorption des Benzylradikals
}

\author{
Von Werner Bingel
}

Aus der Forschungsstelle für Spektroskopie in der Max-Planck-Gesellschaft, Hechingen

(Z. Naturforschg. 10a, 462-476 [1955]; eingegangen am 9. April 1955)

\begin{abstract}
Es werden der Grundzustand und die ersten angeregten Elektronenzustände des Benzylradikals mit der Methode der Valenzstrukturen, der Methode der Molekülbahnen und dem Modell der freien Elektronen berechnet. Die Ergebnisse dieser drei Methoden sind untereinander und mit den spektroskopischen Daten in qualitativer Übereinstimmung. Mit den beiden letzten Methoden werden die Elektronenstruktur des Benzylkations und -anions im Grundzustand und die ersten angeregten Zustände derselben bestimmt.
\end{abstract}

$\mathrm{I}^{\mathrm{n}}$ n neuerer Zeit sind von verschiedener Seite Elektronenbandenspektren beobachtet worden, die dem Benzylradikal zugeordnet wurden 1, 2. Es ist daher von Interesse, dieses Radikal theoretisch mit den Methoden der Quantenchemie zu behandeln. Die Elektronenstruktur des Grundzustandes wurde in der Literatur schon öfters diskutiert ${ }^{3}$. Die angeregten Zustände sind dagegen - so weit uns bekannt ist - noch nicht untersucht worden. Es ist daher der Hauptzweck dieser Arbeit, insbesondere im Hinblick auf die oben erwähnten spektroskopischen Ergebnisse, eine Berechnung dieser angeregten Zustände bezüglich ihrer energetischen Lage relativ zum Grundzustand, ihrer gruppentheoretischen Klassifizierung sowie der Polarisation und Intensität der entsprechenden Elektronen-Übergänge durchzuführen. Da die zur Verfügung stehenden Rechenmethoden alle nur - mehr oder weni-

${ }^{1}$ H. Schüler u. A. Michel, Z. Naturforschg. 10a, 459 [1955], vorangehende Arbeit.

2 G. Porter u. J. Norman, Nature, Lond. 174, 508 [1954]. ger gute - Näherungsverfahren sind, werden wir die Rechnung sowohl mit der Methode der Valenzstrukturen (in § 1), der Methode der Molekülbahnen (in § 2) als auch dem Modell der freien Elektronen (in $\S 3$ ) durchführen. Eine qualitative Übereinstimmung der Ergebnisse dieser drei Verfahren ist dann trotz des approximativen Charakters derselben eine Bestätigung der Zuverlässigkeit dieser Ergebnisse. In $\S 4$ werden wir die erhaltenen Resultate dann untereinander und mit den spektroskopischen Daten vergleichen.

\section{§ 1. Die Methode der Valenzstrukturen}

Der Grundzustand des Benzylradikals ist 1933 von Pauling und Wheland behandelt den $^{3 a}$. Diese Autoren verwendeten die in Abb. 1 dargestellten fünf kanonischen Strukturen für die

3 a) L. Pauling u. G. W. Wheland, J. Chem. Phys. 1, 362 [1933]: Resonanzenergie mit der Methode der Valenzstrukturen. b) W. G. Penney u. G. J. Kynch, Proc. Roy. Soc., Lond. A 164, 409 [1938]: 
Dublett-Terme des Benzylradikals. Mit diesen lautet die Säkulardeterminante für die Gesamtenergie $W$

\begin{tabular}{|c|c|c|c|c|c|}
\hline & $\mathbf{A}$ & B & $\mathbf{E}$ & D & C \\
\hline A & $8 / 8$ & $2 / 11$ & $1 / 7$ & $2 / 8$ & $4 / 10$ \\
\hline B & $2 / 11$ & $8 / 8$ & $4 / 10$ & $2 / 8$ & $1 / 7$ \\
\hline E & $1 / 7$ & $4 / 10$ & $8 / 8$ & $4 / 10$ & $2 / 8$ \\
\hline D & $2 / 8$ & $2 / 8$ & $4 / 10$ & $8 / 8$ & $4 / 10$ \\
\hline $\mathrm{C}$ & $4 / 10$ & $1 / 7$ & $2 / 8$ & $4 / 10$ & $8 / 8$ \\
\hline
\end{tabular}



A

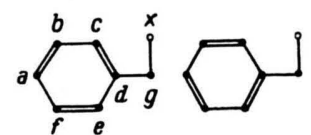

B
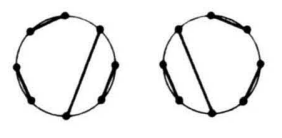

D

C
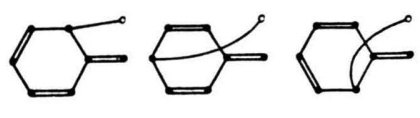

Abb. 1. Die fünf nichtangeregten Strukturen des Benzylradikals. Die obere Reihe gibt die Rumer-Diagramme, die untere Reihe die entsprechenden Strukturen. $x$ ist die Phantombahn ${ }^{4}$, mit der das jeweils unpaarige $\pi$-Elektron verknüpft wird. A und B entsprechen den zwei Kékulé-Strukturen des Benzols, das unpaarige Elektron befindet sich bei dem Methylen CAtom g. In den Strukturen C, E bzw. D ist es in den Ring an die $o$ - bzw. $p$-Position hereingewandert.

Die Elemente dieser Determinante sind dabei

$$
m / n \equiv m(Q-W)+n \cdot \alpha,
$$

wo $W$ die Energie, $Q$ das Coulomb-Integral und $\alpha$ das einfache Austauschintegral zwischen zwei benachbarten $\pi$-Atombahnen ist ${ }^{4}$. Die Bestimmung der Nullstellen von (1) läßt sich durch Ausnutzung der Symmetrien, die das Kohlenstoffgerüst des



Abb. 2. Koordinatensysteme und Symmetrieelemente des C-Gerüstes des Benzylradikals. Die Spiegelebene $\sigma_{v}(x z)$ ist die Papierebene, die Spiegelebene $\sigma_{v}(y z)$ steht senkrecht dazu und geht durch die in der $z$-Richtung liegende zweizählige Drehachse $C_{2}(z) . x_{1},-x_{1}$ und $x_{2}$ beziehen sich auf die Koordinatenwahl bei dem Modell der freien Elektronen.

Atomabstände, berechnet mit den beiden ersten Methoden. c) Disc. Faraday Soc. [1947]: Bindungsordnung und freie Valenz, berechnet mit den beiden ersten Methoden. d) C. A. Coulson, Proc. Roy. Soc., Lond. A 192, 16 [1947]: Die ,nichtbindende“" Molekülbahn und die $\pi$-Elektronenverteilung im Benzylkation und -anion mit der zweiten Methode. e) M. S. J. Dewar,
Benzylradikals besitzt, erleichtern. Wie Abb. 2 zeigt, gehört das Benzylradikal zur Symmetriegruppe $\boldsymbol{C}_{2 v}$, deren Elemente $C_{2}(z)$ (zweizählige Drehachse), $\sigma_{v}(x z)$ und $\sigma_{v}(y z)$ (Spiegelebenen) in dieser Abbildung angegeben sind. Die Charakterentafel für $\boldsymbol{C}_{2 v}{ }^{7}$ ist in Tab. 1 wiedergegeben.

\begin{tabular}{c|rrrr|l}
$C_{2}$ & $I$ & $C_{2}(z)$ & $\sigma_{v}(x z)$ & $\sigma_{v}(y z)$ & \\
\hline $\mathrm{A}_{1}$ & 1 & 1 & 1 & 1 & $z$ \\
$\mathrm{~A}_{2}$ & 1 & 1 & -1 & -1 & \\
$\mathrm{~B}_{1}$ & 1 & -1 & 1 & -1 & $x$ \\
$\mathrm{~B}_{2}$ & 1 & -1 & -1 & 1 & $y$ \\
\hline$\Gamma(\mathrm{A} \ldots \mathrm{E})$ & 5 & -1 & -5 & 1 & \\
$\Gamma(\mathrm{a} \ldots \mathrm{g})$ & 7 & -3 & -7 & +3 & \\
\hline
\end{tabular}

Tab. 1. Die irreduziblen Darstellungen der Symmetriegruppe $C_{2} v$ und ihre Charaktere.

Da die $2 \mathrm{p} \pi$-Atombahnen (AO's) a bis g von Abb. 1 und 2 antisymmetrisch zur Molekülebene $\sigma_{v}(x z)$ sind, und ferner in jeder Struktur sieben, also eine ungerade Zahl, solcher AO's vorkommen, sind alle Strukturen antisymmetrisch $\mathrm{zu}$ dieser Ebene. Die möglichen Molekülzustände müssen daher entweder zur irreduziblen Darstellung $\mathrm{A}_{2}$ oder $B_{2}$ von Tab. 1 gehören. Sie sind dann antisymmetrisch $\left(\mathrm{A}_{2}\right)$ bzw. symmetrisch $\left(\mathrm{B}_{2}\right)$ zur Spiegelebene $\sigma_{v}(y z)$.

Durch die fünf Strukturen A bis $\mathrm{E}$ von Abb. 1 wird eine reduzible Darstellung $\Gamma$ von $\boldsymbol{C}_{2 v}$ induziert, deren Charaktere in der vorletzten Zeile von Tab. 1 angegeben sind und die sich gemäß (3) ausreduzieren läßt.

$$
\Gamma(\mathrm{A} \ldots \mathrm{E})=2 \mathrm{~A}_{2}+3 \mathrm{~B}_{2} .
$$

Man überzeugt sich leicht, daß die folgenden Linearkombinationen bereits die richtige Symmetrie besitzen (die Multiplizität der Terme ist wie üblich durch den linken oberen Index gekennzeichnet):

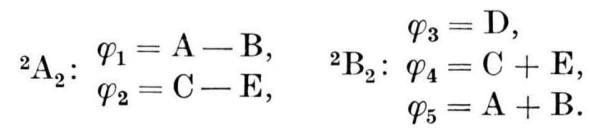

Stellt man daher die Säkulardeterminante (1) mit diesen Funktionen auf, so zerfällt sie in zwei Stufen, eine vom 2. Grade für die zu $\sigma_{v}(y z)$ antisym-

Proc. Camb. Phil. Soc. 45, 638 [1949]: Die Säkulardeterminante für die zweite Methode.

${ }^{4}$ Für eine Erklärung der hier und im folgenden auftretenden Größen und Begriffe siehe etwa ${ }^{5,6}$.

${ }^{5}$ W. Bingel, Z. Naturforschg. 9 a, 436 [1954].

6 W. Bingel, Z. Naturforschg. 9a, 824 [1954].

7 G. Herzberg, Molecular Spectra and Molecular Structure II, New York 1951, S. 106. 
metrischen $\left(\mathrm{A}_{2}\right)$ und eine vom 3. Grade für die zu $\sigma_{v}(y z)$ symmetrischen $\left(\mathrm{B}_{2}\right)$ Molekülzustände ${ }^{8}$.

\begin{tabular}{l|ccccc}
\multicolumn{1}{c}{} & 1 & 2 & 3 & 4 & 5 \\
\cline { 2 - 6 } 1 & $12 /-6$ & $6 / 6$ & 0 & 0 & 0 \\
2 & $6 / 6$ & $12 / 0$ & 0 & 0 & 0 \\
3 & 0 & 0 & $8 / 8$ & $8 / 20$ & $4 / 16$ \\
4 & 0 & 0 & $8 / 20$ & $20 / 32$ & $10 / 34$ \\
5 & 0 & 0 & $4 / 16$ & $10 / 34$ & $20 / 38$
\end{tabular}

Setzt man die zu ${ }^{2} \mathrm{~A}_{2}$ gehörende Stufe gleich Null, so erhält man mit (2) und der Abkürzung $x=(Q-E) / \alpha$ die quadratische Gleichung

$$
\begin{gathered}
3 x^{2}-4 x-1=0, \text { also } \\
x_{1,2}=1 / 3(2 \mp \sqrt{7})=\left\{\begin{array}{l}
-0,21525, \\
+1,54858 .
\end{array}\right.
\end{gathered}
$$

Einsetzen dieser Werte in die Säkulargl. (7)

$$
\begin{gathered}
(12 x-6) C_{1}+(6 x+6) C_{2}=0, \\
(6 x+6) C_{1}+12 x C_{2}=0
\end{gathered}
$$

gibt dann das Verhältnis der Koeffizienten $C_{1}$ und $C_{2}$, mit denen die Symmetriefunktionen $\varphi_{1}$ und $\varphi_{2}$ in die Molekülfunktion eingehen. Man erhält so

$$
\begin{aligned}
& \Psi\left(1^{2} \mathrm{~A}_{2}\right)=(\mathrm{A}-\mathrm{B})+1,82288(\mathrm{C}-\mathrm{E}), \\
& W\left(1^{2} \mathrm{~A}_{2}\right)=Q+0,21525 \alpha, \\
& \Psi\left(2^{2} \mathrm{~A}_{2}\right)=(\mathrm{A}-\mathrm{B})-0,82288(\mathrm{C}-\mathrm{E}), \\
& W\left(2^{2} \mathrm{~A}_{2}\right)=Q-1,54858 \alpha,
\end{aligned}
$$

wo gemäß (4) noch die $\varphi$ 's durch die ursprünglichen Valenzfunktionen A bis $\mathrm{E}$ ersetzt worden sind. Die „Hauptquantenzahl“ vor dem Symmetriecharakter numeriert die nach wachsender Energie geordneten Terme gleicher Symmetrie. Da das Austauschintegral $\alpha$ negativ ist, hat (8a) die tiefere Energie.

Nullsetzen der $B_{2}$-Stufe von (5) führt nach einiger Rechnung auf die kubische Gleichung

$$
x^{3}+1,8 x^{2}-1,8 x-0,8=0 .
$$

Die numerische Lösung derselben ergibt die Nullstellen

$x_{1}=-2,40929, x_{2}=-0,34716_{5}, x_{3}=+0,95645_{5}$.

Zur Bestimmung der Koeffizienten $C_{3}, C_{4}, C_{5}$ setzt man - da es ja nur auf dieVerhältnisse ankommt$\mathrm{C}_{5}=1$ und hat, entsprechend $\mathrm{zu}(7)$,

${ }^{8}$ Der verwendete Satz von fünf Strukturen ist allerdings nicht vollständig. Hierzu müßte man noch weitere acht einfach angeregte und eine zweifach angeregte Struktur berücksichtigen. Man nennt eine Struktur einfach bzw. zweifach angeregt, wenn sie eine bzw. zwei ,lange" Bindungen besitzt, die drei Dewar-Strukturen des Benzols sind in dieser Bezeichnungsweise
$(8 x+8) C_{3}+(8 x+20) C_{4}=-(4 x+16)$,

$(8 x+20) C_{3}+(20 x+32) C_{4}=-(10 x+34)$.

Einsetzen der Nullstellen (9b) in (10) gibt dann, entsprechend $\mathrm{zu}(8)$,

$\Psi\left(1^{2} \mathrm{~B}_{2}\right)=(\mathrm{A}+\mathrm{B})+0,63923(\mathrm{C}+\mathrm{E})+0,60550 \mathrm{D}$, $W\left(1^{2} \mathrm{~B}_{2}\right)=Q+2,40929 \alpha$;

$\Psi\left(2^{2} \mathrm{~B}_{2}\right)=(\mathrm{A}+\mathrm{B})-0,55609(\mathrm{C}+\mathrm{E})-0,96338 \mathrm{D}$,

$W\left(2^{2} \mathrm{~B}_{2}\right)=Q+0,34716_{5} \alpha$;

$\Psi\left(3^{2} \mathrm{~B}_{2}\right)=(\mathrm{A}+\mathrm{B})-3,7496 \quad(\mathrm{C}+\mathrm{E})-4,8577 \quad \mathrm{D}$, $W\left(3^{2} \mathrm{~B}_{2}\right)=Q-0,95645_{5} \alpha$.

Dabei ist (11a) der Grundzustand des Benzylradikals. Es ist von Interesse, (11 a) mit den Ergebnissen von Pauling und Wheland ${ }^{3 a}$ zu vergleichen. Diese Autoren setzten zur Vereinfachung der Rechnung die Koeffizienten $C_{4}$ und $C_{5}$ einander gleich, wodurch an Stelle von $(9 \mathrm{a})$ eine quadratische Gleichung für $x$ tritt, und erhielten so

$\Psi\left(1^{2} \mathrm{~B}_{2}\right)=(\mathrm{A}+\mathrm{B})+0,62579(\mathrm{C}+\mathrm{D}+\mathrm{E})$, $W\left(1^{2} \mathrm{~B}_{2}\right)=Q+2,40914 \alpha$.

Wie man sieht, wird durch diese Vereinfachung die Energie des Grund-Zustandes nur um 1,5 $10^{-4}|\alpha|$ zu hoch bestimmt, was auch darin zum Ausdruck kommt, daß $C_{4}$ und $C_{5}$ in (11 a) angenähert gleich dem Koeffizienten in $\left(11 a^{\prime}\right)$ sind.

Bei Pauling und Wheland steht für diesen Koeffizienten ein falscher Wert von 1,0279; die zweite Wurzel $-0,32770$, die bei Pauling und Wheland nicht angegeben ist, ist eine, allerdings wesentlich schlechtere Näherung für die zweite Wurzel $x_{2}$ von (9a) und damit für den ersten angeregten ${ }^{2} \mathbf{B}_{2}$-Zustand.

Für den in $\S 4$ durchgeführten Vergleich der ersten Absorption bzw. Emission des Benzylradikals mit denen des Benzols benötigen wir noch die Elektronenzustände des letzteren. Beschränkt man sich wie beim Benzylradikal auf nichtangeregte Strukturen, so ist ${ }^{3 \mathrm{a}, 9}$

$$
\begin{aligned}
& \Psi\left({ }^{1} \mathrm{~A}_{1 \mathrm{~g}}\right)=\mathrm{I}+\mathrm{II}, W\left({ }^{1} \mathrm{~A}_{1 \mathrm{~g}}\right)=Q+2,4 \alpha, \\
& \Psi\left({ }^{1} \mathrm{~B}_{2 \mathrm{u}}\right)=\mathrm{I}-\mathrm{II}, W\left({ }^{1} \mathrm{~B}_{2 \mathrm{u}}\right)=Q,
\end{aligned}
$$

wo I und II die beiden Kékulé-Strukturen des Benzols sind; (12a) bzw. (12 b) ist der Grundzustand bzw. der erste angeregte Elektronenzu-

einfach angeregt. Die durch diese insgesamt vierzehn Strukturen induzierte Darstellung gibt ausreduziert $5 A_{2}+9 B_{2}$, man hätte also je eine Gleichung fünften bzw. neunten Grades zu lösen. Aus diesem Grunde haben wir uns ebenso wie Pauling und Wheland auf die nichtangeregten Strukturen $\mathrm{A}$ bis $\mathrm{E}$ beschränkt.

9 A. L. Sklar, J. Chem. Phys. 5, 669 [1937]. 


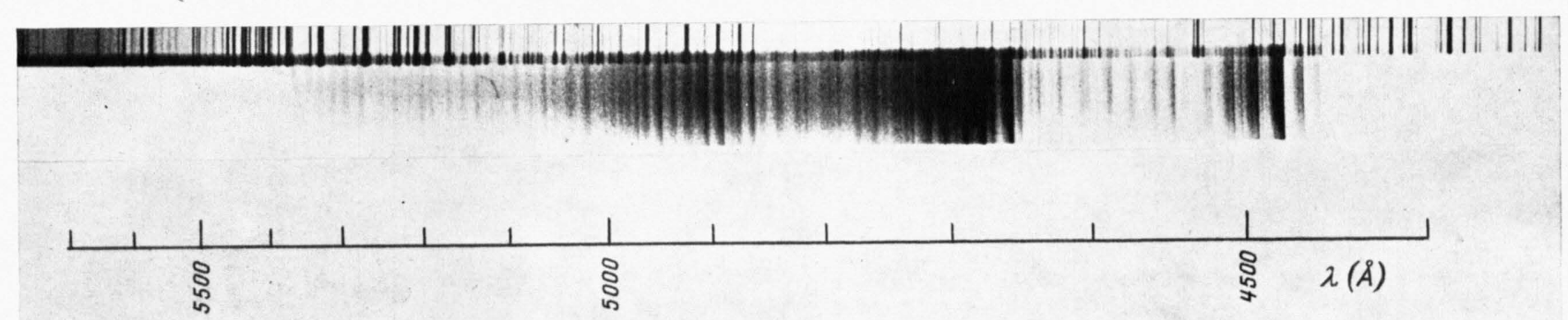

Abb. 1. Spektrum des Benzylradikals (V-Spektrum), beobachtet in der Entladung durch Toluoldampf. Aufgenommen mit Zeiss-Försterling-Spektrograph; $f=27 \mathrm{~cm}, I=5 \mathrm{~mA}, t=30 \mathrm{~min}$.

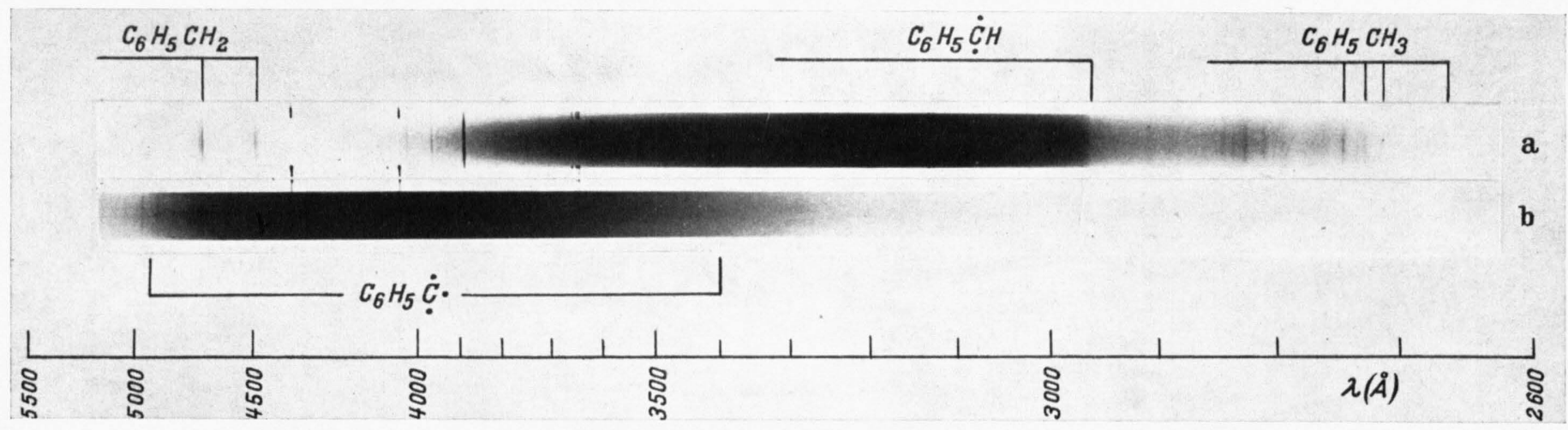

Abb. 2. a) Spektrum des Benzalradikals $\left(\mathrm{C}_{6} \mathrm{H}_{5} \mathrm{CH}\right)$, beobachtet in der Entladung durch Dibenzyldampf. Temperatur des Entladungsraumes $+60^{\circ} \mathrm{C}$. Aufgenommen mit dem mittleren Hilger-Quarzspektrographen; $I=7,5 \mathrm{~mA}, t=15 \mathrm{~min}$. Wesentlich schwächer sind die Spektren des Benzylradikals und des Toluols zu sehen. b) Vermutliches Spektrum des $\mathrm{C}_{6} \mathrm{H}_{5} \mathrm{C}-\mathrm{Radikals}$, beobachtet in der

Entladung durch den Dampf des tertiären Butylbenzols. Temperatur des Entladungsraumes $+250^{\circ} \mathrm{C} ; I=30 \mathrm{~mA}, t=10 \mathrm{~min}$. 


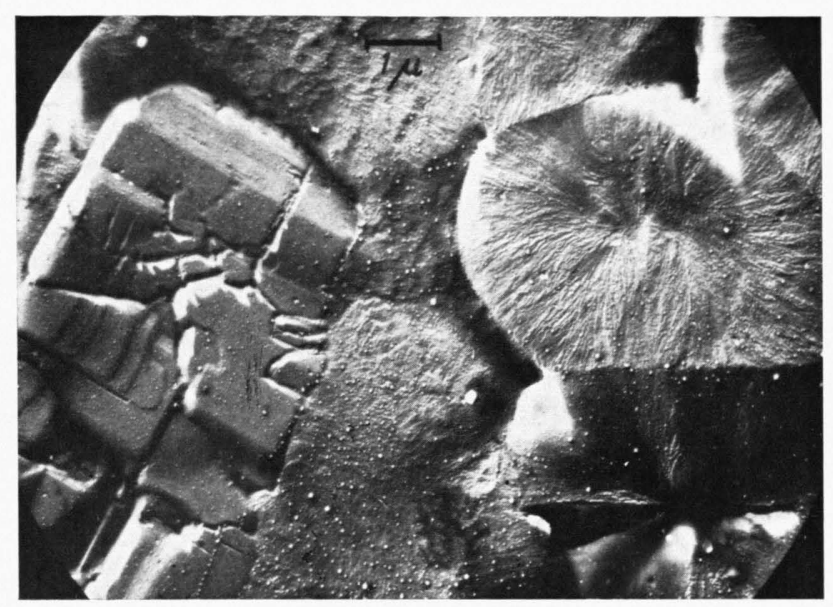

Abb. 1. Vergr.: 10000:1 Sphärolith- und Stäbchenstrukturen des Selens.

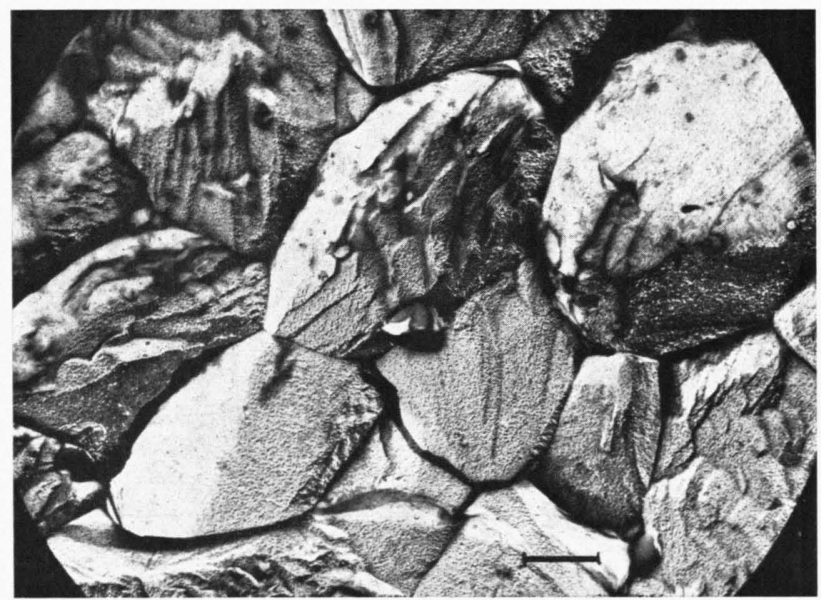

Abb. 3. Vergr. : 10000: 1 Pflastersteinstruktur einer getemperten Selenoberfläche.

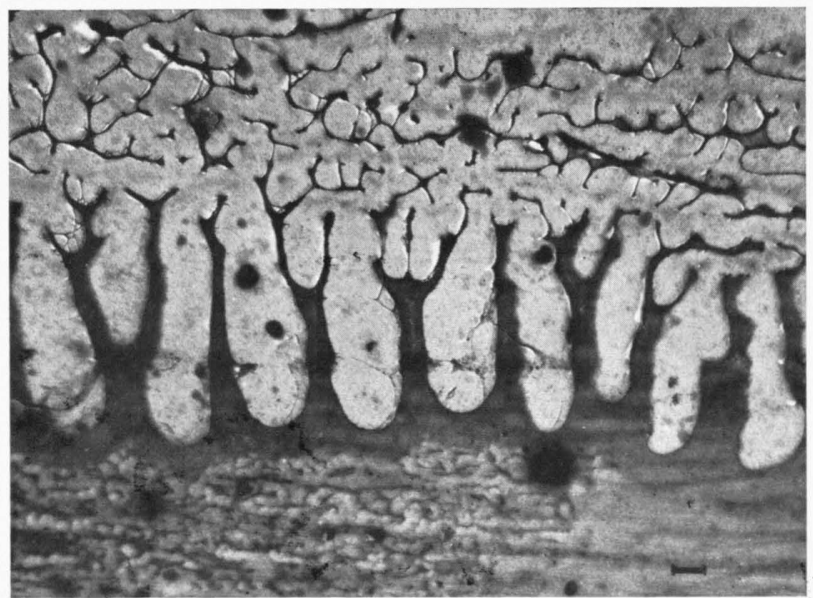

Abb. 5. Vergr. : 4500: 1 Querschnitt eines 30 $\mu$-Kondensats. Trägerplatte am unteren Rande des Bildes. (Nicht sichtbar.)

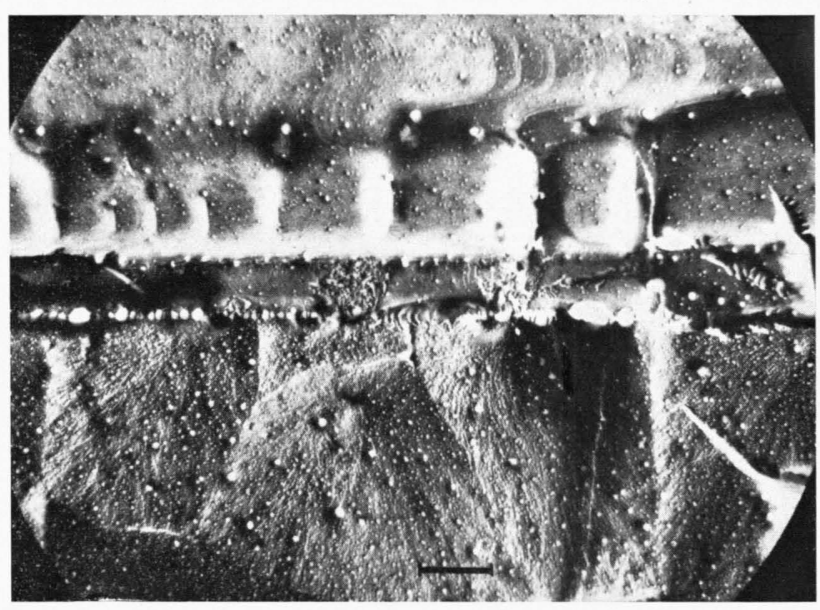

Abb. 2. Vergr.: 10000:1 Selen. Nadelwachstum.

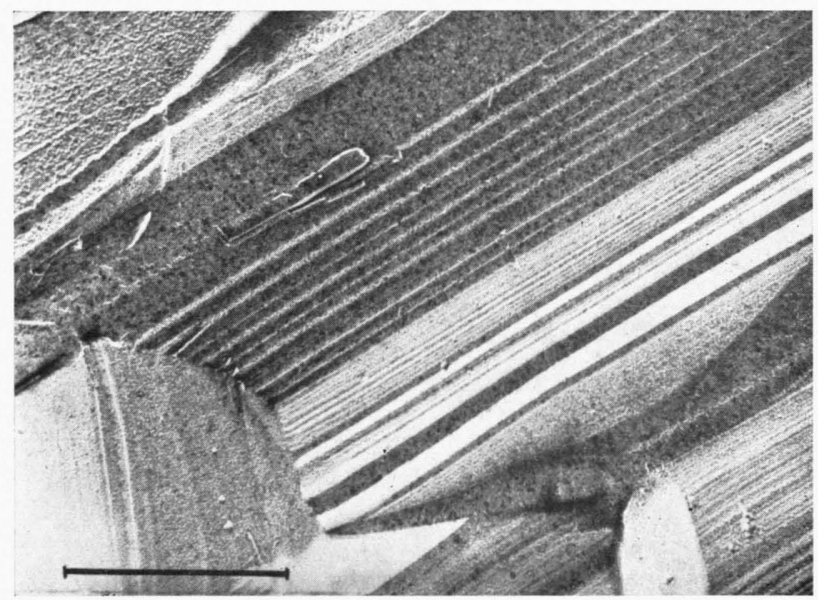

Abb. 4. Vergr.: 30000:1 Plastisches Verhalten des Selens.

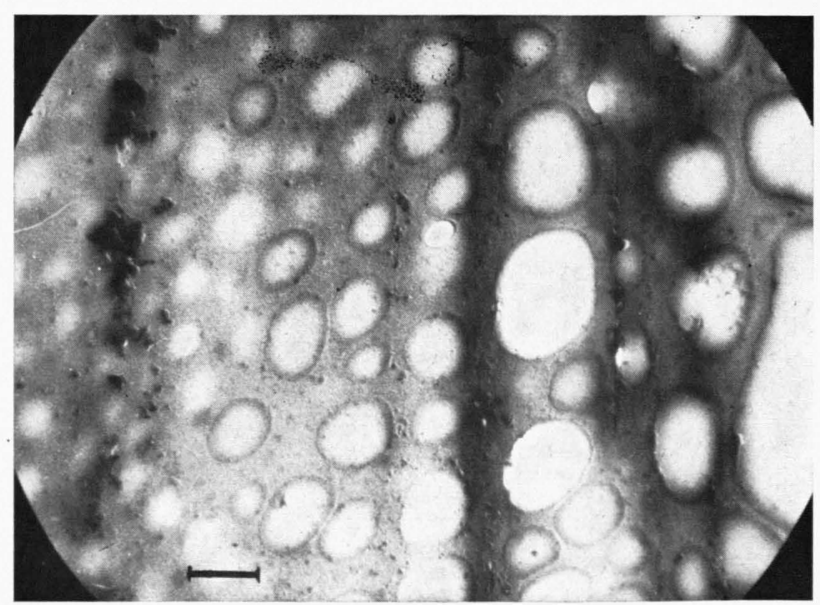

Abb. 6. Vergr.: 10000:1 Querschnitt eines $30 \mu$ Kondensats. Trägerplatte am linken Rande des Bildes. (Nicht sichtbar.) 
stand des Benzols. Bei Mitnahme der drei einfach angeregten Dewar-Strukturen bleibt (12b) ungeändert, während die Energie des Grundzustandes $(12 \mathrm{a})$ jetzt $Q+2,6055 \alpha$ beträgt. In Abb. 3 sind die Terme des Benzols und des Benzylradikals, wie sie sich mit der Methode der Valenzstrukturen ergeben, wiedergegeben. Die erste Absorption des Benzols bei $38000 \mathrm{~cm}^{-1}$ entspricht dem Übergang ${ }^{1} B_{2 u} \leftarrow{ }^{1} A_{1 g}$. Er ist als reiner Elektronenübergang verboten und wird erst durch Mitwirkung einer nichttotalsymmetrischen Schwingung erlaubt. Aus dieser Zahl und der theoretischen Energieänderung von $2,4|\alpha|$ errechnet man einen empirischen Wert von $15830 \mathrm{~cm}^{-1}$ für $|\alpha|$.

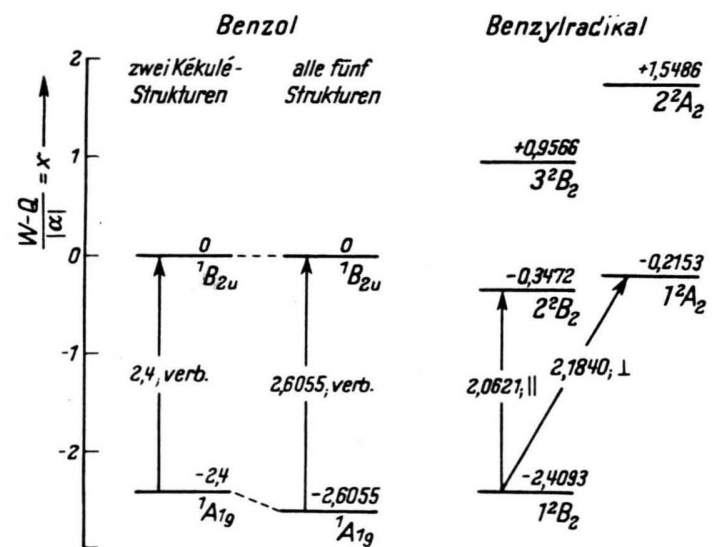

Abb. 3. Termschema des Benzols und des Benzylradikals nach der Methode der Valenzstrukturen. Beim Benzol ist nur der erste angeregte Zustand eingezeichnet. Die Zahlen an den Pfeilen geben die berechnete Energie des betreffenden Übergangs in Einheiten von $|\alpha|$. || und $\perp$ bedeuten zur Symmetrieachse $C_{2}(z)$ parallele bzw. senkrechte Polarisation des betreffenden Übergangs. Beim Benzol ist der erste Übergang verboten.

Die beiden ersten Absorptionen des Benzylradikals sind nach Abb. $32^{2} \mathrm{~B}_{2} \leftarrow \mathrm{l}^{2} \mathrm{~B}_{2}$ und $\mathrm{l}^{2} \mathrm{~A}_{2} \leftarrow \mathrm{l}^{2} \mathrm{~B}_{2}$. Das direkte Produkt der Darstellungen des oberen und unteren Zustandes ist $B_{2} \times B_{2}=A_{1}$ bzw.

$$
\mathrm{B}_{2} \times \mathrm{A}_{2}=\mathrm{B}_{1} \text {. }
$$

Dies gibt bekanntlich die Darstellung der Komponente des Dipolmoments, das mit dem betreffenden Übergang verknüpft ist. Die letzte Spalte von Tab. 1 zeigt, daß der langwelligste Übergang zur $Z$-Komponente des Übergangsmoments gehört, also parallel zur Symmetrieachse polarisiert ist. Der nächste Übergang gehört zur $X$-Komponente und ist daher senkrecht zur Symmetrieachse polarisiert. Nimmt man für $|\alpha|$ den gleichen Wert, wie er oben aus dem Benzolübergang ermittelt wurde, so sollten die beiden erșten Übergänge des Benzylradikals bei etwa 32600 bzw. $34600 \mathrm{~cm}^{-1}$ liegen, also relativ zum ersten Benzolübergang nach Rot verschoben sein.

\section{§ 2. Die Methode der Molekülbahnen}

Bei diesem Verfahren werden aus den $n=7$ $2 \mathrm{p} \pi-\mathrm{AO}$ 's $\chi_{r}(r=1 \ldots n)$, die in Abb. 2 mit a . g bezeichnet worden sind, gewisse Linearkombinationen $\psi_{i}$, eben die ,Molekülbahnen“ (MO's) gebildet:

$$
\psi_{i}=\sum_{r=1}^{n} C_{r i} \chi_{r}
$$

Die Koeffizienten $C_{r i}$ der $r$-ten AO in der $i$-ten MO bestimmen sich nach dem Variationsverfahren aus den Säkulargleichungen

$$
\sum_{(s \neq r)} C_{s i} \beta_{r s}+C_{r i}\left(\alpha_{r}-\varepsilon_{i}\right)=0(i, r=1,2 \ldots n),
$$

dabei ist die Nichtorthogonalität benachbarter AO's vernachlässigt worden. Die $\alpha_{r}$ und $\beta_{r s}$ sind Matrixelemente eines effektiven EinelektronenHamilton-Operators $h$ mit den $\mathrm{AO}^{\prime} \mathrm{s} \chi_{r}$ :

$\alpha_{r}=\int \chi_{r}^{*}(1) h \chi_{r}(1) \mathrm{d} \tau_{1}$ Einelektronen-CoulombIntegral,

$\beta_{r s}=\int \chi_{r}^{*}(1) h \chi_{s}(1) \mathrm{d} \tau_{1}$ Resonanzintegral;

$\varepsilon_{i}$ ist die Energie eines $\pi$-Elektrons in der $i$-ten MO. Die Lösbarkeitsbedingung für das lineare, homogene Gleichungssystem (14) ist das Verschwinden der Säkulardeterminante:

$$
\begin{array}{cccc}
\alpha_{1}-\varepsilon & \beta_{12} & \cdots & \beta_{1 n} \\
\beta_{21} & \alpha_{2}-\varepsilon & & \\
\vdots & \ddots & \vdots \\
\beta_{n 1} & & \cdots & \alpha_{n}-\varepsilon
\end{array}
$$

Einsetzen einer der $n$ Wurzeln $\varepsilon_{i}$ von (16) in (14) gibt die $C_{r i}$ bis auf einen gemeinsamen Faktor, der durch die Normierung festgelegt werden kann:

$$
\sum_{r=1}^{n} C_{r i}^{2}=1,(i=1,2 \ldots n) .
$$

Man vereinfacht (16) durch die für Kohlenwasserstoffe ohne Heteroatome gut zutreffenden Näherungen

$$
\begin{aligned}
& \alpha_{r}=\alpha_{c} \quad \text { für alle } r, \\
& \beta_{r s}= \begin{cases}\beta & \text { für nächste Nachbaratome } r, s, \\
0 & \text { für Nichtnachbarn. }\end{cases}
\end{aligned}
$$


Für das Benzylradikal nimmt (16) dann mit der Abkürzung $y=\left(\alpha_{c}-\varepsilon\right) / \beta$ die Form (19a) an:

\begin{tabular}{l|lllllll}
\multicolumn{1}{l}{} & $\mathrm{g}$ & $\mathrm{d}$ & $\mathrm{e}$ & $\mathrm{f}$ & $\mathrm{a}$ & $\mathrm{b}$ & $\mathrm{c}$ \\
\cline { 2 - 7 } $\mathrm{g}$ & $y$ & 1 & & & & & \\
$\mathrm{~d}$ & 1 & $y$ & 1 & & & & 1 \\
$\mathrm{e}$ & & 1 & $y$ & 1 & & & \\
$\mathrm{f}$ & & & 1 & $y$ & 1 & & \\
$\mathrm{a}$ & & & & 1 & $y$ & 1 & \\
$\mathrm{~b}$ & & & & & 1 & $y$ & 1 \\
$\mathrm{c}$ & & 1 & & & & 1 & $y$
\end{tabular}$=0$.

Man kann zeigen, daß alle MO's zu einer irreduziblen Darstellung der Symmetriegruppe des Moleküls gehören müssen. Welche irreduziblen Darstellungen dabei auftreten, bestimmt man folgendermaßen. Die sieben $2 \mathrm{p} \pi$-AO's a ..g bilden die Basis einer reduziblen Darstellung $\Gamma$, deren Charakterensystem in der letzten Zeile von Tab. 1 angegeben ist. Ausreduzieren gibt ${ }^{10}$

$$
\Gamma(\mathrm{a} \ldots \mathrm{g})=2 \mathrm{a}_{2}+5 \mathrm{~b}_{2} .
$$

Da die MO's gemäß (14) Linearkombinationen von a ...g sind, gibt es also zwei zu $\sigma_{v}(y, z)$ antisymmetrische $\left(\mathrm{a}_{2}\right.$, und fünf zu $\left.\sigma_{v}(y, z)\right)$ symmetrische $\left(b_{2}\right)$ MO's. Man kann von dieser Tatsache Gebrauch machen, indem man die Säkulardeterminante (19a) mit den Symmetriefunktionen

$\varphi_{1}=\mathrm{b}-\mathrm{f}, \varphi_{2}=\mathrm{c}-\mathrm{e}\left(\mathrm{zu} \mathrm{a}_{2}\right)$,

$\varphi_{3}=\mathrm{g}, \varphi_{4}=\mathrm{d}, \varphi_{5}=\mathrm{c}+\mathrm{e}, \varphi=\mathrm{b}+\mathrm{f}, \varphi=\mathrm{a}\left(\mathrm{zu} \mathrm{b}_{2}\right)$

bildet $^{11}$. Man erhält so an Stelle von (19a)

$$
\begin{array}{llllllll}
\multicolumn{1}{l}{1} & 2 & 3 & 4 & 5 & 6 & 7 \\
1 & 2 y & 2 & & & 0 & & \\
2 & 2 & 2 y & & & & & \\
3 & & & y & 1 & & & \\
4 & & & 1 & y & 2 & & \\
5 & 0 & & & 2 & 2 y & 2 & \\
6 & & & & & 2 & 2 y & 2 \\
7 & & & & & & 2 & y
\end{array}=0,
$$

also einmal $(2 y)^{2}-4=0$ oder $y_{1,2}= \pm 1$. Das gibt $\varepsilon\left(\mathbf{l} \mathrm{a}_{2}\right)=\alpha_{\mathrm{c}}+\beta, \quad \psi\left(\mathbf{l} \mathrm{a}_{2}\right)=\frac{1}{2}[\mathrm{~b}-\mathrm{f}+\mathrm{c}-\mathrm{e}]$,

$\varepsilon\left(2 \mathrm{a}_{2}\right)=\alpha_{\mathrm{c}}-\beta, \quad \psi\left(2 \mathrm{a}_{2}\right)=\frac{1}{2}[-\mathrm{b}+\mathrm{f}+\mathrm{c}-\mathrm{e}]$,

wobei die Koeffizienten $C_{r i}$ in der oben beschriebenen Form bestimmt wurden. Da das Resonanzintegral $\beta$ ebenso wie das Austauschintegral $\alpha$ der Methode der Valenzstrukturen negativ ist, gehört (21 a) zur tieferen Energie.

Die zu $b_{2}$ gehörende Stufe läßt sich durch mehrmalige Anwendung des Ränderungssatzes für Determinanten leicht berechnen und führt auf

$4 y\left(y^{4}-6 y^{2}+7\right)=0$, also

$y=0$ und $y= \pm(3 \pm \sqrt{2})^{1 / 2}= \pm 2,1010 ; \pm 1,2599$.

Für die Koeffizienten erhält man die leicht zu verifizierende Beziehung

$N=\left(y^{2}-3\right)^{2}\left(1+y^{2}\right)+2\left(\left(y^{2}-2\right)^{2}+\left(y^{2}+2\right)\right)$,

([g], [d], [c + e], [b+f], [d])

$=N^{-1 / 2}\left(y^{2}-3,-y\left(y^{2}-3\right), y^{2}-2,-y, 2\right),(y \neq 0)$,

wo z.B. [g] der Koeffizient der $\mathrm{AO} g$ ist.

Die durch Einsetzen der $y_{i}$ von (22) in (23) erhaltenen Koeffizienten sind, zusammen mit den aus (22) bestimmten Einelektronenenergien $\varepsilon_{i}$ in Tab. 2 angegeben. Die $\varepsilon_{i}$ sind, wie dies auch schon in (8), (11) und (21) geschehen ist, innerhalb einer irreduziblen Darstellung mit wachsender Energie durchlaufend numeriert, so daß

\begin{tabular}{|c|c|c|c|c|c|c|c|}
\hline \multirow{2}{*}{ MO } & Energie & \multicolumn{6}{|c|}{ Koeffizienten } \\
\hline & $\left(\varepsilon-\alpha_{e}\right) / \beta$ & {$[\mathrm{g}]$} & {$[d]$} & {$[c+e]$} & {$[b+f]$} & {$[\mathrm{a}]$} & Norm.-Faktor $N^{-1 / 2}$ \\
\hline$\frac{1}{5} b_{2}$ & $\pm \sqrt{3+\sqrt{2}}= \pm 2,1010$ & & $6+2 \sqrt{2}$ & $(\sqrt{2}+1)$ & $\pm \sqrt{3+\sqrt{2}}$ & 2 & $\times[8(3+\sqrt{2})]^{-1 / 2}$ \\
\hline${ }_{4}^{2} b_{2}$ & $\pm \sqrt{3-\sqrt{2}}= \pm 1,2599$ & $-\sqrt{2}$ & $6-2 \sqrt{2}$ & $-(\sqrt{2}-1)$ & $=\sqrt{3-\sqrt{2}}$ & 2 & $\times[8(3-\sqrt{2})]^{-1 / 2}$ \\
\hline \multirow[t]{2}{*}{$3 b_{2}$} & $=0,0000$ & 2 & 0 & -1 & 0 & 1 & $\times 7^{-1 / 2}$ \\
\hline & & {$[\mathrm{g}]$} & [d] & {$[c-e]$} & {$[b-f]$} & [a] & Norm.-Faktor $N^{-1 / 2}$ \\
\hline${ }_{2}^{1} \mathrm{a}_{2}$ & \pm 1 & 0 & 0 & 1 & \pm 1 & 0 & $\times 1 / 2$ \\
\hline
\end{tabular}

$$
\varepsilon\left(1 \mathrm{~b}_{2}\right)<\varepsilon\left(2 \mathrm{~b}_{2}\right)<\ldots<\varepsilon\left(5 \mathrm{~b}_{2}\right) \quad \text { ist. }
$$

Tab. 2. Energien und Koeffizienten der MO's des Benzylradikals. Die beiden a ${ }_{2}$ MO's von Gl. (21) sind im unteren Teil der Tabelle nochmals aufgeführt.

10 Für die Symbole der irreduziblen Darstellungen von Ein-Elektronenfunktionen pflegt man die entsprechenden kleinen Buchstaben von Tab. $1 \mathrm{zu}$ verwenden.
$11 \mathrm{Da} \beta$ diese $\varphi$ 's tatsächlich die geforderte Symmetrie besitzen, macht man sich leicht an Hand von Tab. 1 und Abb. 2 unter Berücksichtigung der Antisymmetrie von a ..g bezüglich der Molekülebene $\sigma_{v}(x z)$ klar. 
Eine Betrachtung von Tab. 2 zeigt, daß die MO's in Paaren auftreten, deren beide Partner relativ zu der Energie $\alpha_{c}$ einer isolierten $2 \mathrm{p} \pi-\mathrm{AO}$ entgegengesetzt gleiche Energien und bis auf das Vorzeichen gleiche Koeffizienten haben. Ferner tritt eine ,nichtbindende“ ${ }^{\text {MO }}{ }^{12}$ der Energie Null auf, deren Koeffizienten für diejenigen AO's gleich Null sind, bei denen diese für die MO-Paare entgegengesetztes Vorzeichen haben (ungesternte Atome, s. u.). Diese Eigenschaften gelten allgemein für jeden alternierenden Kohlenwasserstoff mit ungerader Zahl von C-Atomen ${ }^{13}$. Alternierend heißt ein Kohlenwasserstoffmolekül dann, wenn sich seine C-Atome so in zwei Gruppen - eine gesternte und eine ungesternte - einteilen lassen, daß jedes C-Atom aus einer Gruppe als nächste Nachbarn nur solche aus der anderen Gruppe hat. In diesem Fall sind die $\pi$-Ladungsdichten $q_{r}$

$q_{r}=\sum_{i} n_{i} C_{r i}{ }^{2}\left(n_{i}=\right.$ Besetzungszahl der $i$-ten MO)

alle gleich 1 . Die $\pi$-Bindungsordnungen $p_{r s}(24 \mathrm{~b})$ und die freien Valenzen $F_{r}(24 \mathrm{c})$ sind schon früher angegeben worden ${ }^{3 \mathrm{c},}{ }^{4}$ :

$p_{r s}=\sum_{i} n_{i} C_{r i} C_{s i}$,

$F_{r}=N_{\max }-\sum_{s} p_{r s}$ alle nächsten Nachbarn von $r$.

In Abb. 4 sind die MO-Energien $\varepsilon_{i}$ des Benzylradikals nach Tab. 2 denen des Benzols gegenübergestellt. Es sind dies die möglichen Energieniveaus für ein $\pi$-Elektron im Feld des C-Gerüstes und aller anderen $\pi$-Elektronen. Die Gesamtenergie ergibt sich in der gleichen Näherung zu

$$
E=\sum_{i} n_{i} \varepsilon_{i}
$$

das heißt als Summe der Energien der besetzten MO's. Nach dem Pauli-Prinzip kann eine nichtentartete MO maximal $n_{i}=2$ Elektronen aufnehmen. Für den energetisch tiefsten Zustand ist dies in Abb. 4 dargestellt, die kleinen Kreise stellen die $\pi$-Elektronen dar.

Beim Benzol erhält man so die Grundzustandskonfiguration $\left(a_{2 \mathrm{u}}\right)^{2}\left(\mathrm{e}_{1 \mathrm{~g}}\right)^{4}{ }^{1} \mathrm{~A}_{1 \mathrm{~g}}$. Die erste Anregung kommt durch den Übergang eines $\pi$-Elektrons gemäß $\mathrm{e}_{2 \mathrm{u}} \leftarrow \mathrm{e}_{1 \mathrm{~g}}$ zustande. Da die beiden beteiligten

12 Man bezeichnet eine MO mit der Energie

$$
\varepsilon_{i}=\alpha_{c}-y_{i} \beta=\alpha_{c}+y_{i} \cdot|\beta|
$$

als bindend (lockernd), wenn $y_{i}<0(>0)$, als nichtbindend für $y_{i}=0$.
MO's je zweifach entartet sind, ist dieser Übergang mit der Anregungsenergie $2|\beta|$ vierfach entartet (das ist nur die Bahnentartung, die Spinentartung gibt einen weiteren Faktor 4).

Bei Berücksichtigung der Elektronenwechselwirkung ${ }^{14}$ - die durch die Terme $e^{2} / r_{i j}$ in der exakten Hamilton-Funktion hervorgerufen wird spaltet dieser vierfach entartete angeregte Zustand auf gemä $\beta^{15}$

$$
\mathrm{a}_{2 \mathrm{u}}{ }^{2} \mathrm{e}_{1 \mathrm{~g}}{ }^{3} \mathrm{e}_{2 \mathrm{u}}={ }^{1} \mathrm{~B}_{2 \mathrm{u}}+{ }^{1} \mathrm{~B}_{1 \mathrm{u}}+{ }^{1} \mathrm{E}_{1 \mathrm{u}} .
$$

Diese drei Terme, von denen ${ }^{1} \mathrm{E}_{1 \mathrm{u}}$ noch zweifach entartet ist, entsprechen den drei bekannten $\mathrm{Ab}$ sorptionen des Benzols bei 38000, 48000 und $54500 \mathrm{~cm}^{-1}$. Der Schwerpunkt dieser drei Terme liegt bei $48500 \mathrm{~cm}^{-1}$. Setzt man diese aus den experimentellen Daten gewonnene Zahl der theoretisch bestimmten Anregungsenergie nullter Näherung von $2|\beta|$ gleich, so erhält man

$$
|\beta|=24250 \mathrm{~cm}^{-1} \text {. }
$$

Die Elektronenkonfiguration des Benzylradikals im Grundzustand ist aus Abb. 4 zu entnehmen, sie ist $\left(1 b_{2}\right)^{2}\left(2 b_{2}\right)^{2}\left(1 a_{2}\right)^{2} 3 b_{2} l^{2} B_{2}$. Die ersten angeregten Zustände erhält man durch die in Abb. 4 mit Pfeilen markierten Elektronenübergänge, wobei
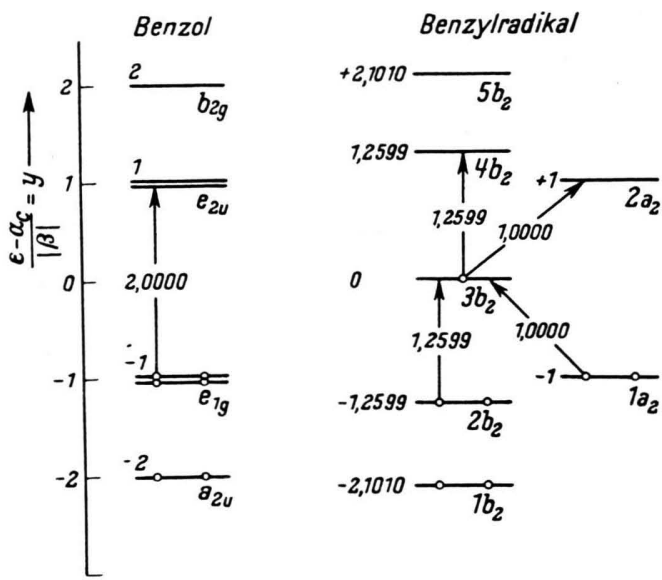

Abb. 4. Schema der MO-Energien beim Benzol und dem Benzylradikal. Die $\circ$ geben die Besetzung der MO's mit Elektronen für den Grundzustand an. Gemäß dem Pauli-Prinzip kann jede nichtentartete MO maximal zwei Elektronen aufnehmen. Beim Benzol sind die MO's bei $\pm \beta$ zweifach entartet, der eingezeichnete Übergang ist also vierfach.

${ }^{13}$ C. A. Coulson u. H. C. Longuet-Higgins, Proc. Roy. Soc., Lond. A 192, 16 [1947].

${ }_{14}$ M. Goeppert-Maier u. A. L. Sklar, J. Chem. Phys. 6, 645 [1938].

${ }^{15}$ Die Spinentartung bedingt, daß jeder dieser drei Terme noch einmal als Triplett auftritt. 
$3 b_{2} \leftarrow 1 a_{2}$ und $2 a_{2} \leftarrow 3 b_{2}$ zur gleichen Anregungsenergie von $l|\beta|$ gehören, also miteinander entartet sind. Ebenso gehören $3 b_{2} \leftarrow 2 b_{2}$ und $4 b_{2} \leftarrow 3 b_{2}$ zur gleichen Anregungsenergie von 1,2599 | $\beta$ | und sind miteinander entartet. Durch Berücksichtigung der Elektronenwechselwirkung spalten die miteinander entarteten Zustände auf, so daß man die in Abb. 5 wiedergegebenen Terme des Benzylradikals erhält. $\mathrm{Zm}$ Vergleich sind in Abb. 5 auch die oben besprochenen Terme des Benzols eingezeichnet. Die angegebenen Termbezeichnungen und Polarisationsrichtungen folgen aus

$\left.\begin{array}{l}2 \mathrm{a}_{2} \leftarrow 3 \mathrm{~b}_{2}: \ldots\left(1 \mathrm{a}_{2}\right)^{2} 2 \mathrm{a}_{2} \\ 3 \mathrm{~b}_{2} \leftarrow 1 \mathrm{a}_{2} \ldots 1 \mathrm{a}_{2}\left(3 \mathrm{~b}_{2}\right)^{2}\end{array}\right\}=1^{2} \mathrm{~A}_{2}+2^{2} \mathrm{~A}_{2}$

$\mathrm{B}_{2} \times \mathrm{A}_{2}=\mathrm{B}_{1}$, also $X$-Polarisation;

$\left.\begin{array}{l}4 b_{2} \leftarrow 3 b_{2}: \ldots\left(1 a_{2}\right)^{2} 4 b_{2} \\ 3 b_{2} \leftarrow 2 b_{2}: 2 b_{2}\left(1 a_{2}\right)^{2}\left(3 b_{2}\right)^{2}\end{array}\right\}=2^{2} B_{2}+3^{2} B_{2}$,

$\mathrm{B}_{2} \times \mathrm{B}_{2}=\mathrm{A}_{1}$, also $Z$-Polarisation. $\left[\mathrm{cm}^{-1}\right.$ ]


Abb. 5. Termschema des Benzols, des Benzylradikals und des Benzylkations bzw. -anions nach der Methode der Molekülbahnen. Die Zahlen an den Pfeilen bedeuten beim Benzol die beobachteten, beim Benzylradikal die berechneten Oszillatorstärken $f$, bei den Benzoltermen handelt es sich um experimentelle Werte.

${ }^{16}$ M. J. S. Dewar u. H. C. Longuet-Higgins, Proc. Phys. Soc., Lond. A 67, 795 [1954].
Die Aufspaltungen können aus dieser nullten Näherung natürlich nicht entnommen werden, wir werden sie im folgenden noch bestimmen.

Nach der Methode der Molekülbahnen erwartet man also für das Benzylradikal vier langwellige Übergänge, von denen zwei senkrecht, und zwei parallel zur Symmetrieachse $C_{2}(z)$ polarisiert sind. Das Mittel aus den beiden senkrecht polarisierten Übergängen sollte bei der doppelten Wellenlänge wie der Schwerpunkt der drei ersten Benzolübergänge liegen.

In diesem Zusammenhang ist eine Arbeit von Dewar und Longuet-Higgins von Interesse ${ }^{16}$, in der die beobachteten langwelligsten Absorptionen (die $\alpha, \mathrm{p}, \beta$ und $\beta^{\prime}$-Banden von Clar) von alternierenden Kohlenwasserstoffen und Kohlenwasserstoffradikalen mittels der Methode der Molekülbahnen mit teilweiser Berücksichtigung der Elektronenwechselwirkung gedeutet werden. Insbesondere zeigen diese Autoren für alternierende Radikale, daß durch Aufspaltung entstehende Terme 
wie $1^{2} \mathrm{~A}_{2}, 2^{2} \mathrm{~A}_{2}$ und $2^{2} \mathrm{~B}_{2}, 3^{2} \mathrm{~B}_{2}$ in (26) (siehe auch den mittleren Teil von Abb. 5) so beschaffen sind, daß der energetisch tiefere ein sehr kleines (in ihrer Näherung sogar Null), der energetisch höhere ein relativ viel größeres Übergangsmoment vom Grundzustand aus besitzt.

Wir wollen die allgemeinen Formeln dieser Autoren benutzen, um für das Benzylradikal die Übergangsmomente und die beiden Aufspaltungen zu berechnen. Hierzu bildet man zunächst gemäß (26) die folgenden antisymmetrisierten Gesamteigenfunktionen in der Form von Slater-Determinanten:

$$
\begin{aligned}
\chi_{0}\left(1^{2} \mathrm{~B}_{2}\right) & =\left|1 b_{2} \overline{1 b_{2}} 2 b_{2} \overline{2 b_{2}} 1 a_{2} \overline{1 a_{2}} 3 b_{2}\right|, \\
\chi_{1}\left(2 a_{2} \leftarrow 3 b_{2}\right) & =\left|1 b_{2} \overline{1 b_{2}} 2 b_{2} 2 b_{2} 1 a_{2} 1 a_{2} 2 a_{2}\right|, \\
\chi_{2}\left(3 b_{2} \leftarrow 1 a_{2}\right) & =\left|11 b_{2} \overline{1 b_{2}} 2 b_{2} 2 b_{2} 1 a_{2} 3 b_{2} 3 b_{2}\right|
\end{aligned}
$$

und entsprechend für $\chi_{1}\left(4 b_{2} \leftarrow 3 b_{2}\right), \chi_{2}\left(3 b_{2} \leftarrow 2 a_{2}\right)$. Wie schon oben erwähnt, sind $\chi_{1}$ und $\chi_{2}$ miteinander entartet. Bei Berücksichtigung ihrer Wechselwirkung erhält man an ihrer Stelle die Funktionen $^{16}$

$\Psi_{1,2}=2^{-1 / 2}\left(\chi_{1} \pm \chi_{2}\right)$ zur Energie $E=E^{0} \pm \gamma$.

Dabei ist $E^{0}$ die ungestörte Energie, die nach dem oben Gesagten gleich $1 \cdot|\beta|$ für die $\mathrm{A}_{2}$-Terme und $1,2599|\beta|$ für die $\mathrm{B}_{2}$-Terme ist. Das Abstoßungsintegral $\gamma$ ist gegeben durch $^{16}$

$$
\gamma=-\iint \varrho(1) \cdot \frac{1}{r_{12}} \varrho(2) \mathrm{d} \tau_{1} \mathrm{~d} \tau_{2}, \quad \text { wo }
$$

$\varrho(1)=\sum_{r} * C_{r}\left(2 \mathrm{a}_{2}\right) C_{r}\left(3 \mathrm{~b}_{2}\right) \cdot \chi_{r}{ }^{2}(1)$ für die $\mathrm{A}_{2}$-Terme

und entsprechend für die $\mathrm{B}_{2}$-Terme.

Die Summation läuft dabei nur über die gesternten AO's a, c, e und g, ihre Koeffizienten entnimmt man aus Tab. 2. Man erhält so für die $\mathrm{A}_{2}$ Terme

$$
\begin{aligned}
\varrho(1) & =\frac{1}{2 \sqrt{7}}\left(\mathrm{e}^{2}(1)-\mathrm{c}^{2}(1)\right), \\
\gamma & =-\frac{1}{4 \cdot 7}\left(\gamma_{\mathrm{ee}}+\gamma_{\mathrm{cc}}-2 \gamma_{\mathrm{ec}}\right)=-\frac{1}{14}\left(\gamma_{11}-\gamma_{13}\right) .
\end{aligned}
$$

Dabei sind die Coulomb-Integrale $\gamma_{\mu \nu}$ definiert durch

$$
\gamma_{\mu \nu}=\int\left\lceil\chi_{\mu}{ }^{2}(1) \cdot \frac{1}{r_{12}} \cdot \chi_{\nu}{ }^{2}(2) \mathrm{d} \tau_{1} \mathrm{~d} \tau_{2}\right.
$$

und hängen nur von dem Abstand der AO's $\chi_{\mu}$ und $\chi_{\nu}$ ab. Es ist also, wovon wir noch Gebrauch machen werden,

17 J.A.Pople, Proc. Phys. Soc., Lond. A68,81 [1955]. 18 J. A. Pople, Trans. Faraday Soc. 49, 1375 [1953].

$$
\begin{aligned}
& \gamma_{\mathrm{aa}}=\gamma_{\mathrm{bb}}=\ldots=\gamma_{11}=10,53 \mathrm{eV}, \\
& \gamma_{\mathrm{ab}}=\gamma_{\mathrm{bc}}=\ldots=\gamma_{12}=7,30 \mathrm{eV}, \\
& \gamma_{\mathrm{ac}}=\gamma_{\mathrm{ce}}=\ldots=\gamma_{13}=5,46 \mathrm{eV}, \\
& \gamma_{\mathrm{ag}}=3,46 \mathrm{eV} .
\end{aligned}
$$

Die numerischen Werte sind einer Arbeit von Pople ${ }^{17}$ entnommen, mit Ausnahme von $\gamma_{\mathrm{ag}}$, das nach der Näherung der reziproken Abstände ${ }^{18}$ berechnet wurde. Hiermit wird $\gamma\left(\mathrm{A}_{2}\right)=-0,36_{2} \mathrm{eV}$ $=-2920 \mathrm{~cm}^{-1}$. Für die $B_{2}$-Terme wird entsprechend

$$
\begin{aligned}
& \varrho(1)=\frac{1}{\sqrt{56 \cdot(3-\sqrt{2})}} \\
& \cdot\left\{2 \mathrm{a}^{2}(1)+(\sqrt{2}-1)\left(\mathrm{c}^{2}(1)+\mathrm{e}^{2}(1)\right)-2 \sqrt{2} \mathrm{~g}^{2}(1)\right\} .
\end{aligned}
$$

Einsetzen in $\gamma$ gibt nach Zusammenfassen gleicher Glieder

$$
\begin{aligned}
\gamma\left(\mathrm{B}_{2}\right) & =-\frac{1}{56(3-\sqrt{2})} \\
& \cdot\left((18-4 \sqrt{2}) \gamma_{11}-6(\sqrt{2}-1)^{2} \gamma_{13}-8 \sqrt{2} \gamma_{\mathrm{ag}}\right) \\
& =-0,846 \mathrm{eV}=-6820 \mathrm{~cm}^{-1} .
\end{aligned}
$$

Mit den so bestimmten Aufspaltungen sind in Abb. 5 die vier angeregten Terme des Benzylradikals gezeichnet worden. Wir bemerken noch, daß für beide Symmetrien $\gamma<0$ ist, so daß die Funktion $\Psi_{1}$ in (27) energetisch tiefer liegt als ihr Partner $\Psi_{2}$.

Wir wollen ferner noch die Oszillatorstärken der Elektronenübergänge im Benzylradikal vom Grundzustand $\mathrm{l}^{2} \mathrm{~B}_{2}$ zu den vier angeregten $\mathrm{Zu}$ ständen berechnen. Die Oszillatorstärke $f_{\mathrm{B} \leftarrow \mathrm{A}}$ eines Übergangs $\mathrm{B} \leftarrow \mathrm{A}$ bestimmt sich aus dem Übergangsmoment $\boldsymbol{Q}_{\mathrm{B} \leftarrow \mathrm{A}}$ gemä $\boldsymbol{\beta}^{19}$

$$
\begin{aligned}
& f_{\mathrm{B} \leftarrow \mathrm{A}}=\frac{8 m \pi^{2} c}{3 h} \cdot v_{\mathrm{B} \leftarrow \mathrm{A}} \cdot\left|\boldsymbol{Q}_{\mathrm{B} \leftarrow \mathrm{A}}\right|^{2} \\
& =1,08_{5} \cdot 10^{11} \imath_{\mathrm{B} \leftarrow \mathrm{A}} \cdot\left|\boldsymbol{Q}_{\mathrm{B} \leftarrow \mathrm{A}}\right|^{2}, \\
& \boldsymbol{Q}_{\mathrm{B} \leftarrow \mathrm{A}}=\int \ldots \int \Psi_{\mathrm{B}} * \sum_{i} \mathfrak{r}_{i} \Psi_{\mathrm{A}} \mathrm{d} \tau_{1} \ldots \mathrm{d} \tau_{n} . \quad(31 \mathrm{a}, \mathrm{b})
\end{aligned}
$$

Dabei ist $v_{\mathrm{B} \leftarrow \mathrm{A}}$ die Wellenzahl des Übergangs in $\mathrm{cm}^{-1}, \Psi_{\mathrm{A}}$ und $\Psi_{\mathrm{B}}$ sind die Gesamt-Eigenfunktionen des unteren und oberen Zustandes, und die Summation in $(31 \mathrm{~b})$ ist über die Ortsvektoren $\mathfrak{r}_{i}$ aller Elektronen zu erstrecken. Der Koordinatenursprung für die $\mathfrak{r}_{i}$ ist beliebig, sofern nur $\Psi_{\mathrm{A}}$ und $\Psi_{\mathrm{B}}$ zueinander orthogonal sind ${ }^{20}$. Auf Grund der Auswahlregeln (26) ist jeweils nur eine Komponente des Vektors $\boldsymbol{Q}$ von Null verschieden.

19 R. S. Mulliken, Rep. Progr. Phys. 8, 231 [1941].

${ }^{20}$ H. Shull, J. Chem. Phys. 19, 1610 [1951]. 
Setzt man in (31b) die entsprechenden antisymmetrisierten Gesamteigenfunktionen ein, so reduziert sich $\boldsymbol{Q}$ auf die folgenden Einelektronenübergangsmomente für die $\mathrm{A}_{2}$-Terme:

$$
\boldsymbol{Q}_{1,0}=-\boldsymbol{Q}_{2,0}=\underset{r}{\Sigma^{*}} C_{r}\left(2 \mathrm{a}_{2}\right) C_{r}\left(3 \mathrm{~b}_{2}\right) \cdot \mathrm{r}_{r}
$$

und entsprechend für die $B_{2}$-Terme. Die Summation ist dabei wie in (28) nur über die ,,gesternten“ AO's $\mathrm{a}$, c, e und $\mathrm{g} \mathrm{zu}$ erstrecken, $\mathrm{r}_{r}$ ist der Ortsvektor des $r$-ten C-Atoms relativ zum Ursprung, als den wir das Atom a von Abb. 2 wählen. Mit den Koeffizienten $C_{r}$ von Tab. 2 erhält man sofort

$$
\boldsymbol{Q}_{1,0}=-\boldsymbol{Q}_{2,0}=\frac{1}{2 \sqrt{7}}\left(\mathrm{r}_{e}-\mathrm{r}_{c}\right) .
$$

Aus Abb. 2 ersieht man sofort, daß dies nur eine $X$ Komponente von der Größe

$$
X_{1,0}=-X_{2,0}=-\frac{a}{2} \sqrt{\frac{3}{7}}
$$

hat, $a=1,39 \AA$ ist der für alle C-Atome als gleich angenommene $\mathbf{C}-\mathbf{C}$-Abstand. Mit diesem Wert wird schließlich $X_{1,0}=-X_{2,0}=-0,4550 \AA$. Einsetzen in (31 a) gibt die Oszillatorstärke für die beiden miteinander entarteten Übergänge

$$
f_{2 \mathrm{a}_{2} \leftarrow 3 \mathrm{~b}_{2}}=f_{3 \mathrm{~b}_{2} \leftarrow 1 \mathrm{a}_{2}}=0,0710 .
$$

Auf dem gleichen Wege erhält man für die beiden anderen Übergänge

$Q_{1,0}=-Q_{2,0}=\frac{1}{\sqrt{56(3-\sqrt{3})}}$

$$
\cdot\left((\sqrt{2}-1)\left(\mathrm{r}_{c}+\mathrm{r}_{e}\right)-2 \sqrt{2} \mathrm{r}_{g}\right) \text {. }
$$

Dieses Moment hat in Übereinstimmung mit (26) nur eine $Z$-Komponente der Größe

$$
Z_{1,0}=-Z_{2,0}=-\frac{\sqrt{2}+1}{\sqrt{56(3-\sqrt{3})}} \cdot 3 a=-1,068 \AA .
$$

Die Oszillatorstärke folgt dann aus (31 a) zu

$$
f_{4 \mathrm{~b}_{2} \leftarrow 3 \mathrm{~b}_{2}}=f_{3 \mathrm{~b}_{2} \leftarrow 2 \mathrm{~b}_{2}}=0,378 .
$$

Ohne Berücksichtigung der Elektronenwechselwirkung haben also die beiden miteinander entarteten Terme $\left(\mathrm{A}_{2}\right.$ oder $\left.\mathrm{B}_{2}\right)$ entgegengesetzt gleiche Momente und gleich große Oszillatorstärken. Die Berücksichtigung der Wechselwirkung zwischen den entarteten Zuständen führt zu den Funktionen (27). Offenbar ist nun wegen $\boldsymbol{Q}_{2,0}=-\boldsymbol{Q}_{1,0}$

$$
\begin{aligned}
& Q\left(\Psi_{\left.1, \chi_{0}\right)}=\frac{1}{\sqrt{2}}\left(Q_{1,0}+Q_{2,0}\right)=0,\right. \\
& Q\left(\Psi_{2, \chi_{0}}\right)=\frac{1}{\sqrt{2}}\left(Q_{1,0}-Q_{2,0}\right)=\sqrt{2} Q_{1,0}
\end{aligned}
$$

und damit

$$
\begin{aligned}
& f_{1^{2} \mathrm{~A}_{2} \leftarrow 1^{2} \mathrm{~B}_{2}}=0, \\
& f_{2^{2} \mathrm{~A}_{2} \leftarrow 1^{2} \mathrm{~B}_{2}}=2 f_{2 \mathrm{a}_{2} \leftarrow 3 \mathrm{~b}_{2}}=0,14, \\
& f_{2^{2} \mathrm{~B}_{2} \leftarrow 1^{2} \mathrm{~B}_{2}}=0, \\
& f_{3^{2} \mathrm{~B}_{3} \leftarrow 2^{2} \mathrm{~B}_{3}}=2 f_{4 \mathrm{~b}_{3} \leftarrow 3 \mathrm{~b}_{3}}=0,76 .
\end{aligned}
$$

Es ist anzunehmen, daß eine genauere Rechnung auch für $1^{2} \mathrm{~A}_{2}$ und $2^{2} \mathrm{~B}_{2}$ auf Kosten der $2^{2} \mathrm{~A}_{2}$ - und $3{ }^{2} \mathrm{~B}_{2}$-Terme von Null verschiedene, wenn auch kleine Oszillatorstärken ergeben würde ${ }^{21}$. Die Summe der Oszillatorstärken für die $\mathrm{A}_{2}$ - bzw. $\mathrm{B}_{2}$ Terme muß jedoch ungeändert gleich $0,14 \mathrm{bzw}$. 0,76 bleiben. Die so bestimmten $f$-Werte sind in Abb. 5 an die Pfeile für die entsprechenden U̇bergänge angeschrieben. Zum Vergleich sind dort beim Benzol die experimentell bestimmten $f$-Werte ebenfalls aufgenommen.

In Absorption wird man also nur die folgenden Übergänge

$$
\begin{aligned}
& 2^{2} \mathrm{~A}_{2} \leftarrow 1^{2} \mathrm{~B}_{2}, v=27200 \mathrm{~cm}^{-1} \\
& 3^{2} \mathrm{~B}_{2} \leftarrow 1^{2} \mathrm{~B}_{2}, v=37400 \mathrm{~cm}^{-1}
\end{aligned}
$$

stark sehen. Davon wird der letztere allerdings wohl von der ersten Toluolabsorption bei 38000 $\mathrm{cm}^{-1}$ überdeckt werden.

Wir können hier auch gleich noch das Benzylkation bzw. -anion mitbehandeln. Die MO's und ihre Energie sind natürlich die gleichen, wie beim Benzylradikal, da sich ja das Kohlenstoffgerüst nicht geändert hat. Was sich für den Grundzustand ändert, ist lediglich die Besetzungszahl $n$ der nichtbindenden MO $3 \mathrm{~b}_{2}$, die beim Benzylkation null, beim Anion zwei und beim Radikal eins beträgt.
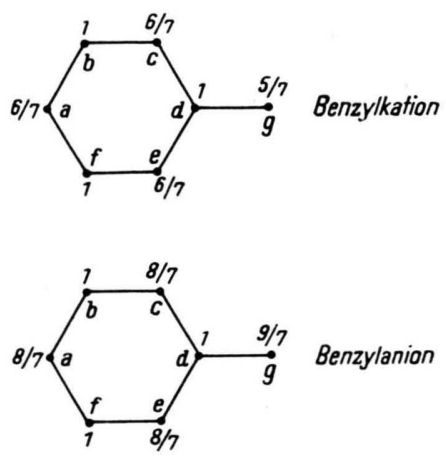

Abb. 6. Die $\pi$-Ladungsdichten $q_{r}$ von Gl. (34) im Benzylkation und -anion.

Da für das Radikal die $\pi$-Ladungsdichte gleich eins ist (s. o.), folgt hieraus sofort ${ }^{3 \mathrm{~d}}$, daß wegen der Definition (24a)

$$
\begin{aligned}
& q_{r}(\text { Kation })=1-C_{r}^{2}\left(3 \mathrm{~b}_{2}\right) \text { und } \\
& q_{r}(\text { Anion })=1+C_{r}^{2}\left(3 \mathrm{~b}_{2}\right),
\end{aligned}
$$

beide sind in Abb. 6 wiedergegeben.

${ }^{21}$ In Abb. 5 ist dies dadurch zum Ausdruck gebracht, daß die $f$-Werte des jeweils schwächeren Übergangs mit $f \ll 0,14$ bzw. $f \ll 0,76$ angegeben sind. 
Dabei sind die $C_{r}\left(3 \mathrm{~b}_{2}\right)$ die Koeffizienten der $r$ ten $\mathrm{AO}$ in der nichtbindenden $\mathrm{MO} 3 \mathrm{~b}_{2}$. Wegen des Verschwindens dieser Koeffizienten für die ungesternten C-Atome trägt diese MO nichts zur Bindungsordnung $(24 \mathrm{~b})$ und damit auch nichts zur freien Valenz (24c) bei. Diese Größen haben daher in den beiden Ionen die gleichen Werte wie im Ra$d_{i k a l}^{3 \mathrm{~d}}$.

Gemäß dem oben Gesagten ist die Elektronenkonfiguration für den Grundzustand $\left(1 \mathrm{~b}_{2}\right)^{2}\left(2 \mathrm{~b}_{2}\right)^{2}$ $\left(1 a_{2}\right)^{2}{ }^{1} A_{1}$ für das Kation, $\left(1 b_{2}\right)^{2}\left(2 b_{2}\right)^{2}\left(1 a_{2}\right)^{2}\left(3 b_{2}\right)^{2}$ ${ }^{1} \mathrm{~A}_{1}$ für das Anion. Die ersten angeregten Zustände erhält man durch die Elektronenübergänge

$$
\begin{aligned}
\text { Kation: } & 3 \mathrm{~b}_{2} \leftarrow 1 \mathrm{a}_{2}={ }^{1} \mathrm{~B}_{1},{ }^{3} \mathrm{~B}_{1} \\
& 3 \mathrm{~b}_{2} \leftarrow 2 \mathrm{~b}_{2}={ }^{1} \mathrm{~A}_{1},{ }^{3} \mathrm{~A}_{1}, \\
\text { Anion: } & 2 \mathrm{a}_{2} \leftarrow 3 \mathrm{~b}_{2}={ }^{1} \mathrm{~B}_{1},{ }^{3} \mathrm{~B}_{1} \\
& 4 \mathrm{~b}_{2} \leftarrow 3 \mathrm{~b}_{2}={ }^{1} \mathrm{~A}_{1},{ }^{3} \mathrm{~A}_{1} .
\end{aligned}
$$

Die Art und Zahl der ersten angeregten Elektronenzustände ist nach (35) für Kation und Anion die gleiche. Da die Anregung der beiden Triplettzustände vom Singulett-Grundzustand aus wegen des Interkombinationsverbots mit nur sehr geringer Intensität erfolgen kann, wird man daher bei Absorptions- oder Emissionsuntersuchungen nur die Übergänge

${ }^{1} \mathrm{~B}_{1} \leftarrow{ }^{1} \mathrm{~A}_{1}, \quad v>24500 \mathrm{~cm}^{-1}, \quad$ Polarisation $X$, ${ }^{1} \mathrm{~A}_{1} \leftarrow{ }^{1} \mathrm{~A}_{1}, \quad v>30550 \mathrm{~cm}^{-1}$, Polarisation $Z$ (36)

stark sehen. Ein Vergleich von (36) mit (33 c) zeigt, daß es unter Umständen schwierig sein wird, zwischen diesen drei Möglichkeiten auf Grund spektroskopischer Daten allein zu unterscheiden.

\section{§ 3. Das Modell der freien Elektronen (FEM)}

Diese Methode nimmt an, daß sich die $\pi$-Elektronen frei in dem durch das Kohlenstoffgerüst von Abb. 2 gegebenen eindimensionalen und verzweigten Raume bewegen können. Sie ist also eine Annäherung an die zweite Methode, da sich die $\pi$ Elektronen in einem konstanten Potential (das man zu Null normieren kann) bewegen sollen, während in jener - wenigstens im Prinzip - mit dem durch die einfach positiven C-Ionen und die restlichen $\pi$-Elektronen erzeugten effektiven Potential gerechnet wird.

Die Lösungen der Schrödinger-Gleichung sind die einfachen Funktionen der schwingenden Saite (konstantes Potential!)

$$
\psi(x)=A \sin k x+B \cos k x,
$$

wo $x$ der längs des C-Gerüstes von einem beliebigen Nullpunkt aus gerechnete Abstand ist; $k$ ist die Wellenzahl, die mit der Energie $\varepsilon$ gemäß $(37 \mathrm{~b})$ zusammenhängt $(\varkappa=k a / \pi)$ :

$\varepsilon=\frac{\hbar^{2}}{2 m} k^{2}=\frac{h^{2}}{8 m a^{2}} \cdot \varkappa^{2}=1,569_{6} \cdot 10^{5} \varkappa^{2}\left[\mathrm{~cm}^{-1}\right]$,

das letztere für einen C-C-Abstand von $a=1,39 \AA$.

Für den bei uns vorliegenden Fall von Verzweigungen hat man für jeden Verzweigungspunkt $\mathbf{P}$ die folgenden Anschlußbedingungen zu erfüllen, die die Funktionen $\psi\left(x_{i}\right)$ der in $\mathrm{P}$ zusammenlaufenden $Z$ weige $i$ miteinander verknüpfen:

$\left.\psi\left(x_{1}\right)\right|_{\mathbf{P}}=\left.\psi\left(x_{2}\right)\right|_{\mathbf{P}}=\ldots ;\left.\quad \sum_{i} \frac{\partial \psi\left(x_{i}\right)}{\partial x_{i}}\right|_{\mathbf{P}}=0$.

$(38 \mathrm{a}, \mathrm{b})$

Um am Ende eines Seitenzweiges nicht identisch verschwindende Ladungsdichte $\mathrm{zu}$ bekommen, denkt man sich jeden Seitenzweig um einen C-CAbstand verlängert und setzt dann $\psi$ an diesem neuen Ende E gleich Null (in Abb. 2 ist dies durch die gestrichelte Fortsetzung über g hinaus zum Ausdruck gebracht):

$$
\left.\psi(x)\right|_{\mathbf{E}}=0 .
$$

Man kann nun so vorgehen, daß man für jeden Zweig $i$ eine Lösung von der Form (37a) ansetzt, die aus $(38 \mathrm{a}-\mathrm{c})$ folgenden Gleichungen legen dann die Koeffizienten $A_{i}, B_{i}$ bis auf einen gemeinsamen Faktor fest, außerdem erhält man eine transzendente Gleichung für die Wellenzahl $k$, deren Lösungen dann mit ( $37 \mathrm{~b}$ ) die Energieniveaus $\varepsilon$ geben.

Zur Beschreibung des Benzylradikals benötigen wir die in Abb. 2 eingezeichneten zwei Koordinaten $x_{1}$ und $x_{2}$ (die Koordinate des dritten $Z$ weiges ist $-x_{1}$ ), für die Verzweigung $\mathrm{P}$ gilt dann $x_{1}=3 a$, $x_{2}=2 a$ und für den Endpunkt E $x_{2}=0$. Entsprechend dem oben Gesagten machen wir den Ansatz

$$
\begin{aligned}
\psi_{1} & =\left\{\begin{array}{l}
A \sin k x_{1} \\
B \cos k x_{1}
\end{array},\right. \\
\psi_{2} & =\left\{\begin{array}{c}
0 \quad\left(\text { antisymmetr. zu } \sigma_{\nu}(y z)\right) \\
C \sin k x_{2}\left(\text { symmetr. zu } \sigma_{\nu}(y z)\right) .
\end{array}\right.
\end{aligned}
$$

Hierbei sind gegenüber (37a) die Endbedingung (38c) und die Symmetrie bezüglich der Spiegelebene $\sigma_{\nu}(y z)$ bereits berücksichtigt. Wir haben daher nur noch die Verzweigungsbedingung $(38 \mathrm{a}, \mathrm{b})$ zu erfüllen. 
Antisymmetrischer Fall:

Aus (38a) folgt $A \sin 3 k a=0,(38 b)$ ist identisch erfüllt, also ist

$$
\varkappa_{n}^{(\mathrm{a})}=\frac{k a}{\pi}=\frac{n}{3}, \quad n=1,2 \ldots .
$$

$$
\text { Symmetrischer Fall: }
$$

Aus (38a, b) erhält man die Bedingungsgleichungen $B \cos 3 k a=C \sin 2 k a$, $k(-2 B \sin 3 k a+C \cos 2 k a)=0$.

Eine Lösung dieser Gleichungen ist offenbar

$\varkappa_{n}^{(\mathrm{s})}=n+1 / 2, \quad n=0,1 \ldots, \quad C / B=2(-1)^{n}$.

Wenn wir diese Lösung im folgenden ausschließen, können wir die Bedingungsgleichungen umformen in

$$
2 \operatorname{tg} 3 k a=\operatorname{cotg} 2 k a, \quad \frac{B}{C}=\frac{\sin 2 k a}{\cos 3 k a} .
$$

Mit $t=\operatorname{tg}^{2} k a$ läßt sich diese transzendente Gleichung auf die quadratische Gleichung

$$
7 t^{2}-16 t+1=0, \quad t=\frac{1}{7}(8 \pm \sqrt{57})
$$

reduzieren. Hieraus erhält man wegen $\operatorname{tg}^{2} k a=t$

$$
\begin{gathered}
\sin k a=\frac{1}{2}\left(\frac{9 \pm \sqrt{57}}{6}\right)^{1 / 2}=0,830407 ; 0,24581, \\
\varkappa^{(\mathrm{s})=} \frac{k a}{\pi}=\left\{\begin{array}{l}
0,311892+2 n ;-0,311892+(2 n+1) \\
0,079055+2 n ;-0,079055+(2 n+1)
\end{array}\right. \\
=\frac{B}{C}\left\{\begin{array}{l}
-0,94456 \\
+0,64832
\end{array}, n=0,1,2 \ldots .\right.
\end{gathered}
$$

In Tab. 3 sind die ersten sieben Eigenwerte $\varkappa$ nach (40), (41) und (43) zusammen mit den nach $(37 \mathrm{~b})$ berechneten Energien $\varepsilon$ wiedergegeben. Die

\begin{tabular}{|c|c|c|c|}
\hline FEMO & $x^{(\mathrm{s})} \quad x^{(\mathrm{a})}$ & $\left(\varkappa^{(\mathrm{s})}\right)^{2} \quad\left(\varkappa^{(\mathrm{a})}\right)^{2}$ & $\varepsilon\left[\mathrm{cm}^{-1}\right]$ \\
\hline $1 \mathrm{~b}_{2}$ & 0,07905 & 0,00625 & 981 \\
\hline $2 b_{2}$ & 0,31189 & 0,09727 & 15270 \\
\hline $1 a_{2}$ & & $1 / 9$ & 17440 \\
\hline $3 \mathrm{~b}_{2}$ & 0,5 & 0,25 & 39240 \\
\hline $2 a_{2}$ & & & 69760 \\
\hline $4 b_{2}$ & 0,68811 & 0,47350 & 74320 \\
\hline $5 b_{2}$ & 0,92095 & 0,84815 & 133100 \\
\hline
\end{tabular}
Molekülbahnen des Modells der freien Elektronen (FEMO's) sind dabei - wie die MO's - mit den irreduziblen Darstellungen $\mathrm{b}_{2}$ [symmetrisch $\mathrm{zu}$ $\left.\sigma_{v}(y z)\right]$ und $\mathrm{a}_{2}$ [antisymmetrisch zu $\sigma_{v}(y z)$ ] bezeichnet, innerhalb jeder Gruppe sind sie mit wachsender Energie durchlaufend numeriert.

Tab. 3. Die Energien der ersten sieben FEMO's.

In Abb. 7 sind die ersten sieben FEMO's in einem Energieschema wiedergegeben, um sie mit der entsprechenden Darstellung der MO's in Abb. 4 (rechte Hälfte) vergleichen zu können. Die kleinen Kreise geben wieder die Besetzung der

FEMO's für den Grundzustand des Benzylradikals an. Bei einem Vergleich zwischen Abb. 4 (MO's) und Abb. 7 (FEMO's) sieht man, daß beide Methoden in der Reihenfolge der Molekülbahnen völlig übereinstimmen, auch die Abstände sind für die tieferliegenden Molekülbahnen ähnlich, für die höherliegenden gibt das Modell der freien Elektronen größere Abstände als die Methode der Molekülbahnen. Das liegt daran, daß bei der letzteren die Anzahl der MO's endlich, nämlich gleich der Zahl $n=7$ der zur Verfügung stehenden $2 \mathrm{p} \pi$-AO's ist,

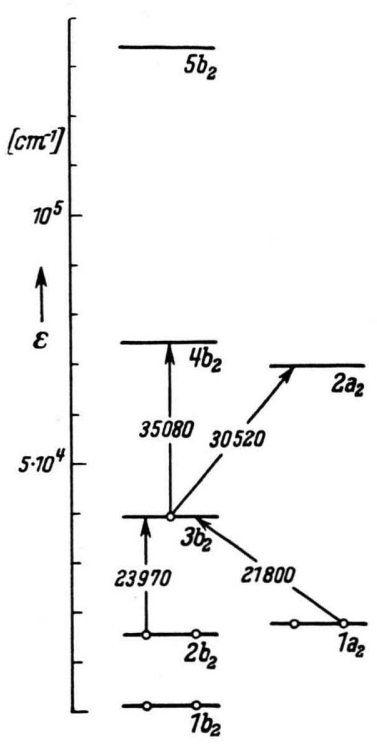

Abb. 7. Schema der FEMO-Energien im Benzylradikal. Im übrigenvgl. die Unterschrift zu Abb. 4. Anregungsenergien in $\mathrm{cm}^{-1}$. In Abb. 8 sind diese angeregten Elektronenzustände des Benzylradikals zusammen mit dessen Grundzustand in einem Termschema für die nach $(24 \mathrm{~d})$ berechneten $G e$. samt-Energien vereinigt, zusammen mit der Symmetriebezeichnung der Terme, der Polarisation und der Oszillatorstärke $f$ (s. unten) der betreffenden Übergänge.

Wie in $\S 2$ so kann man auch hier das Benzylkation bzw. -anion leicht mitbehandeln. Im Kation bzw. Anion ist die FEMO $3 b_{2}$, die der ,nichtbindenden" MO $3 \mathrm{~b}_{2}$ von Abb. 4 entspricht, unbesetzt bzw. doppelt besetzt. Die ersten Übergänge sind wieder durch (35) gegeben. Im Gegensatz zur Methode der Molekülbahnen geben dieselben für das Kation und Anion nicht mehr die gleiche Energie, da hier die symmetrische Lage der MO's relativ 
zur nichtbindenden MO $3 \mathrm{~b}_{2}$ fortfällt. Die entsprechenden Energien lassen sich aus Tab. 3 entnehmen. Sie wurden benutzt, um die in Abb. 8 ebenfalls wiedergegebenen Terme des Benzylkations bzw. -anions zu zeichnen. Wie in $\S 2$ läßt sich auch hier die Singulett-Triplett-Aufspaltung nicht ohne weiteres berechnen.

Schließlich wollen wir noch die Oszillatorstärken $f$ der langwelligsten Übergänge des Benzylradikals mit dem Modell der freien Elektronen berechnen. Für den bei uns vorliegenden speziellen Fall eines Radikals, bei dem im Grundzustand und in den betrachteten angeregten Zuständen alle FEMO's bis auf eine doppelt besetzt sind, vereinfacht (31 b) sich zu

$$
\boldsymbol{Q}_{\mathrm{b} \leftarrow \mathrm{a}}=\int \psi_{\mathrm{b}} * \mathfrak{r} \psi_{\mathrm{a}} \mathrm{d} \tau .
$$

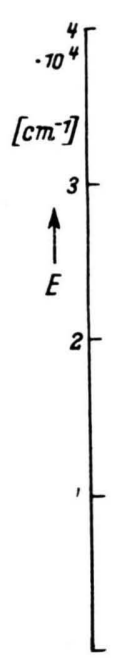

$$
\text { Benzylradikal }
$$

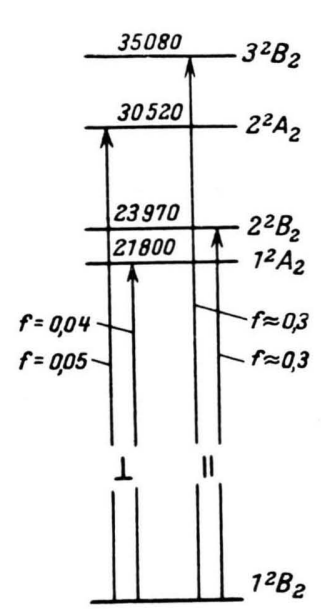

$\psi_{1}\left(3 \mathrm{~b}_{2}\right)=\frac{1}{\sqrt{7 a}} \cos \frac{\pi}{2} \frac{x_{1}}{a}, \quad \psi_{2}\left(3 \mathrm{~b}_{2}\right)=\frac{2}{\sqrt{7 a}} \sin \frac{\pi}{2} \frac{x_{2}}{a}$,

$\psi_{1}\left(2 \mathrm{~b}_{2}\right)=\frac{-1}{\sqrt{4,12085 a}} \cos 0,31189 \pi \frac{x_{1}}{a}$,

$\psi_{2}\left(2 b_{2}\right)=\frac{1}{\sqrt{3,67655 a}} \sin 0,31189 \pi \frac{x_{2}}{a}, \quad(45 \mathrm{a}-\mathrm{e})$

$\psi_{1}\left(4 b_{2}\right)=\frac{-1}{\sqrt{4,12085 a}} \cos 0,68811 \pi \frac{x_{1}}{a}$,

$\psi_{2}\left(4 b_{2}\right)=\frac{1}{\sqrt{3,67655 a}} \sin 0,68811 \pi \frac{x_{2}}{a}$.

Die Absolutwerte der Koeffizienten $A, B, C$ in (39) sind dabei durch die Normierungsbedingung

$$
\int|\psi|^{2} \mathrm{~d} \tau \stackrel{+}{=} \int_{-3 a}^{3 a}\left|\psi_{1}\right|^{2} \mathrm{~d} x_{1}+\int_{0}^{2 a}\left|\psi_{2}\right|^{2} \mathrm{~d} x_{2}=1
$$

festgelegt, aus der sich
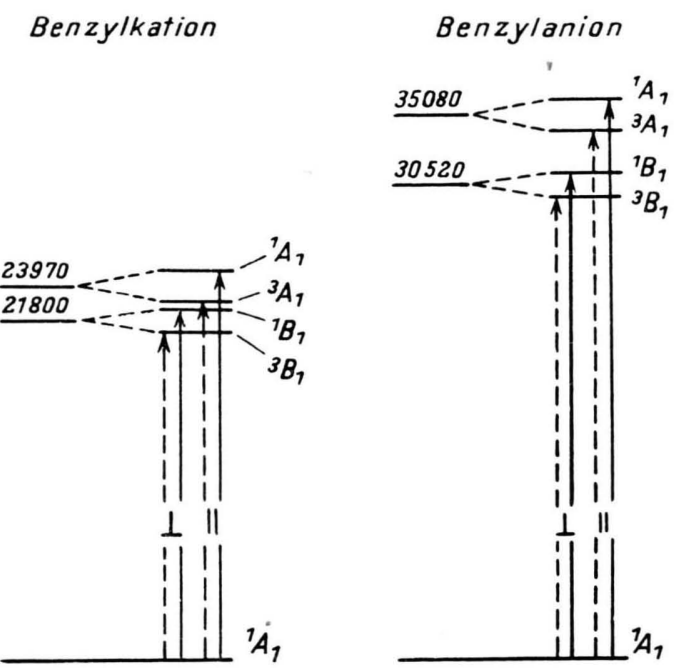

Abb. 8. Termschema des Benzylradikals, des Benzylkations und -anions nach dem Modell der freien Elektronen.

Die Zahlen an den Übergangspfeilen beim Benzylradikal sind die berechneten Oszillatorstärken.

Das ist im Gegensatz zu (31 b) ein Ein-Elektronenintegral, $\psi_{\mathrm{a}}$ und $\psi_{\mathrm{b}}$ sind die Einelektronenfunktionen der im Grundzustand bzw. im angeregten $\mathrm{Zu}$ stand einfach besetzten FEMO's. Es entspricht dies dem Normalfall bei den Atomspektren, daß nur ein Leuchtelektron einen ,,Sprung“ in eine andere Bahn vornimmt.

Die benötigten $\psi_{\mathrm{a}}$ und $\psi_{\mathrm{b}}$ sind nach Abb. 7, Gl. (26) $\psi\left(1 \mathrm{a}_{2}\right), \psi\left(2 \mathrm{a}_{2}\right), \psi\left(2 \mathrm{~b}_{2}\right), \psi\left(3 \mathrm{~b}_{2}\right)$ und $\psi\left(4 \mathrm{~b}_{2}\right)$. Aus (39) bis (43) erhalten wir hierfür

$$
\begin{array}{ll}
\psi_{1}\left(1 \mathrm{a}_{2}\right)=\frac{1}{\sqrt{3 a}} \sin \frac{\pi}{3} \frac{x_{1}}{a}, & \psi_{2}\left(1 \mathrm{a}_{2}\right)=0, \\
\psi_{1}\left(2 \mathrm{a}_{2}\right)=\frac{1}{\sqrt{3 a}} \sin \frac{2 \pi}{3} \frac{x_{1}}{a}, & \psi_{2}\left(2 \mathrm{a}_{2}\right)=0,
\end{array}
$$

$\frac{1}{2} A^{2} \cdot 6 a=1$, also $A=(3 a)^{-1 / 2}$ für $\mathrm{a}_{2}$ und

$\frac{1}{2}\left(B^{2} \cdot 6 a+C^{2} \cdot 2 a\right)=1$,

$C=\left[a\left(1+3\left(\frac{B}{C}\right)^{2}\right)\right]^{-1 / 2}, \quad B=\frac{B}{C} \cdot C \quad$ für $\mathrm{b}_{2}$ ergibt.

Das Verhältnis $B / C$ ist schon durch die Anschlußbedingungen (38) bestimmt und in (41) und (43) angegeben.

Für die beiden Übergänge $3 b_{2} \leftarrow 1 a_{2}$ und $2 a_{2} \leftarrow 3 b_{2}$ von Abb. 7 ist nach (26) nur die $X$-Komponente von (44) von Null verschieden und für $3 b_{2} \leftarrow 2 b_{2}$ und $4 b_{2} \leftarrow 3 b_{2}$ nur die $Z$-Komponente. Aus Abb. 2 entnimmt man leicht, daß folgendes gilt: 


$$
\begin{array}{rlrl}
X & =\frac{\sqrt{3}}{2} x_{1}, & Z & =\frac{1}{2} x_{1} \text { auf a b, } \\
X & =\frac{\sqrt{3}}{2} a, & Z & =x_{1}-\frac{a}{2} \text { auf } \mathrm{b} \mathrm{c}, \\
X & =\frac{\sqrt{3}}{2}\left(3 a-x_{1}\right), Z=\frac{1}{2}\left(a+x_{1}\right) \text { auf c d, } \\
X & =0, & Z & =4 a-x_{2} \text { auf } \mathrm{dg} \mathrm{E} .
\end{array}
$$

Die Lage des Koordinatennullpunkts (in a) ist für die Berechnung des Übergangsmoments (38c) belanglos, wenn nur die $\psi_{\mathrm{a}}, \psi_{\mathrm{b}}$ zueinander orthogonal sind ${ }^{20}$, das ist bei uns der Fall. Mit (45) und (47) erhält man so für den Übergang $3 b_{2} \leftarrow 1 a_{2}$

$$
\begin{aligned}
& X_{3 \mathrm{~b}_{2} \leftarrow 1 \mathrm{a}_{2}}=\underset{\text { abcd }}{2} \psi\left(3 \mathrm{~b}_{2}\right) X \psi\left(1 \mathrm{a}_{2}\right) \mathrm{d} \tau \\
& =\frac{2}{\sqrt{3 a} \cdot \overline{7 a}} \int_{0}^{3 \mathrm{a}} \cos \frac{\pi}{2} \frac{x_{1}}{a} \cdot X \cdot \sin \frac{\pi}{3} \frac{x_{1}}{a} \mathrm{~d} x_{1} .
\end{aligned}
$$

Dabei ist der Anteil des Weges dgE (siehe Abb. 2) gleich Null wegen (45a), das Stück afed gibt den gleichen Wert wie abed und ist durch den Faktor 2 vor dem ersten Integral berücksichtigt. Zerlegen des zweiten Integrals in die drei den Abschnitten ab, bc und cd entsprechenden Anteile gibt wegen (47) mit $\xi=x_{1} / a$

$$
\begin{aligned}
X_{3 \mathrm{~b}_{2} \leftarrow 1 \mathrm{a}_{2}}= & \frac{a}{\sqrt{7}}\left\{\int_{0}^{1} \cos \frac{\pi}{2} \xi \cdot \xi \cdot \sin \frac{\pi}{3} \xi \mathrm{d} \xi\right. \\
& +\int_{1}^{2} \cos \frac{\pi}{2} \xi \cdot \sin \frac{\pi}{3} \xi \mathrm{d} \xi \\
& \left.+\int_{2}^{3} \cos \frac{\pi}{2} \xi \cdot(3-\xi) \cdot \sin \frac{\pi}{3} \xi \mathrm{d} \xi\right\} .
\end{aligned}
$$

Mit $a=1,39 \AA$ gibt die numerische Auswertung der Integrale $X_{3 b_{2} \leftarrow 1 a_{2}}=-0,40303 \AA$. Einsetzen dieses Wertes in (38a) gibt mit $\imath_{3 \mathrm{~b}_{2} \leftarrow 1 \mathrm{a}_{2}}=21800$ $\mathrm{cm}^{-1}$ (Tab. 3), $f_{3 \mathrm{~b}_{2} \leftarrow 1 \mathrm{a}_{2}}=0,038$. Auf die gleiche Weise erhält man für den zweiten $X$-polarisierten Übergang $f_{3 \mathrm{~b}_{2} \leftarrow 1 \mathrm{a}_{2}}=0,047$.

Für die beiden $Z$-polarisierten Übergänge ist die Berechnung der Oszillatorstärke wesentlich umständlicher, da jetzt auch die Strecke dgE beiträgt und die $\psi$-Funktionen (45d,e) nichtrationale Koeffizienten und $x$-Werte haben. Man hat zunächst wieder

$$
\begin{aligned}
& Z_{3 \mathrm{~b}_{2} \leftarrow 2 \mathrm{~b}_{2}=2 \int \psi_{\mathrm{abcd}}\left(3 \mathrm{~b}_{2}\right) \cdot Z \cdot \psi_{1}\left(2 \mathrm{~b}_{2}\right) \mathrm{d} \tau}+\int_{\mathrm{dg} \mathrm{E}} \psi_{2}\left(3 \mathrm{~b}_{2}\right) \cdot Z \cdot \psi_{2}\left(2 \mathrm{~b}_{2}\right) \mathrm{d} \tau= \\
& -2 \sqrt{\frac{1}{7 a \cdot 4,12085 a} \int_{0}^{3 a} \cos \frac{\pi}{2} \frac{x_{1}}{a} \cdot Z \cdot \cos 0,31189 \pi \frac{x_{1}}{a} \mathrm{~d} x_{1}} \\
& +2 \sqrt{\frac{1}{7 a \cdot 3,67655 a} \int_{0}^{a} \sin \frac{\pi}{2} \frac{x_{2}}{a} \cdot Z \cdot \sin 0,31189 \pi \frac{x_{2}}{a} \mathrm{~d} x_{2} .}
\end{aligned}
$$

Zerlegt man das erste Integral wie oben in seine drei Anteile, so erhält man mit $\xi=x / a$

$$
\begin{aligned}
& Z_{3 \mathrm{~b}_{2} \leftarrow 2 \mathrm{~b}_{2}=}-\frac{2 a}{\sqrt{28,846}}\left\{\int_{0}^{1} \cos \frac{\pi}{2} \xi \cdot \frac{\xi}{2} \cdot \cos 0,31189 \pi \xi \mathrm{d} \xi\right. \\
&+\int_{1_{1}^{2}}^{2} \cos \frac{\pi}{2} \xi\left(\xi-\frac{1}{2}\right) \cos 0,31189 \pi \xi \mathrm{d} \xi \\
&\left.+\int_{2}^{3} \cos \frac{\pi}{2} \xi \cdot \frac{\xi+1}{2} \cdot \cos 0,31189 \pi \xi \mathrm{d} \xi\right\} \\
&+\frac{2 a}{\sqrt{25,736}} \int_{0}^{2} \sin \frac{\pi}{2} \xi(4-\xi) \sin 0,31189 \pi \xi \mathrm{d} \xi .
\end{aligned}
$$

Für eine grobe Abschätzung wurden diese Integrale graphisch ausgewertet; man erhält schließlich $f_{3 \mathrm{~b}_{2} \leftarrow 2 \mathrm{~b}_{2}} \approx 0,3$ und etwa den gleichen Wert für $f_{4 \mathrm{~b}_{2} \leftarrow 3 \mathrm{~b}_{2}}$. Daß diese Werte wesentlich größer sind als für die $X$-polarisierten Übergänge, ergibt sich qualitativ schon daraus, daß zu ihnen das Stück $\mathrm{dgE}$ einen großen Beitrag liefert, nämlich das vierte Integral in (51), das bei den $X$-polarisierten Übergängen aus Symmetriegründen fehlen muß. Die so bestimmten $f$-Werte sind in Abb. 8 an die entsprechenden Übergänge angeschrieben.

In Absorption sollte man nur die beiden starken Übergänge

sehen.

$$
\begin{aligned}
& 2{ }^{2} \mathrm{~B}_{2} \leftarrow 1{ }^{2} \mathrm{~B}_{2}, v=23990 \mathrm{~cm}^{-1}, \\
& 3{ }^{2} \mathrm{~B}_{2} \leftarrow 1{ }^{2} \mathrm{~B}_{2}, v=35080 \mathrm{~cm}^{-1}
\end{aligned}
$$

\section{§ 4. Diskussion}

Alle drei Methoden stimmen darin überein, daß der Grundzustand ein ${ }^{2} \mathrm{~B}_{2}$-Term ist und daß die vier ersten angeregten Elektronenzustände je zwei ${ }^{2} \mathrm{~B}_{2}$ - und zwei ${ }^{2} \mathrm{~A}_{2}$-Terme sind. Die Methode der Molekülbahnen (Abb. 5) und das Modell der freien Elektronen (Abb. 8) stimmen auch noch in der Reihenfolge und der ungefähren energetischen Lage der angeregten Terme überein, während die Methode der Valenzstrukturen (Abb. 3) eine andere Reihenfolge ergibt. Hierbei ist jedoch zu beachten, daß wir dort nur nichtangeregte Strukturen berücksichtigt haben. Die Mitnahme der restlichen neun angeregten Strukturen ${ }^{8}$ könnte dies sehr wohl ändern.

Die Polarisation der Übergänge ist durch den Symmetriecharakter der angeregten Terme bestimmt und daher in allen drei Methoden die gleiche. 
Die Intensitäten bzw. die dazu proportionalen Oszillatorstärken konnten nur mit der Methode der Molekülbahnen und dem Modell der freien Elektronen (Abb. 5 und 8) ermittelt werden. Bei einem Vergleich beider ist nun zu beachten, daß die in Abb. 5 und Abb. 8 angegebenen Terme sich in dem Grad der verwendeten Näherung unterscheiden. Bei der MO-Methode sind die $A_{2}$ - und $B_{2}$-Terme miteinander entartet, wenn man die Elektronenwechselwirkung völlig vernachlässigt (nullte Näherung). In der gleichen Näherung sind diese Terme bei dem Modell der freien Elektronen bereits getrennt. Ein Vergleich der Oszillatorstärken $f$ nach diesen beiden Methoden in nullter Näherung zeigt nun eine recht gute Übereinstimmung derselben. Die beiden $\mathrm{A}_{2}$-Terme haben nach der MO-Methode beide ein $f$ von 0,07 , nach dem Modell der freien Elektronen $f$-Werte von 0,04 bzw. 0,05. Die beiden $\mathrm{B}_{2}$-Terme haben, wiederum in beiden Methoden, wesentlich größere Oszillatorstärken, nämlich $f=0,38$ nach der MO-Methode und $f \approx 0,3$ nach dem Modell der freien Elektronen.

Die teilweise Berücksichtigung der Elektronenwechselwirkung in der MO-Methode (erste Näherung) verändert diese $f$-Werte nun in der in $\S 2$ beschriebenen Weise derart, daß der jeweils energetisch höhere Term eines in nullter Näherung entarteten Paares seine Oszillatorstärke auf Kosten seines energetisch tiefer liegenden Partners vergrößert. Ob diese Änderung allerdings so weit geht, wie es durch (33) beschrieben wird, wo der tiefer liegende Term $f=0$ und der höher liegende das doppelte des Wertes der nullten Näherung (32) hat, ist fraglich. Das wirkliche Verhalten dürfte wohl zwischen den durch Gl. (32) und (33) ausgedrückten Tatbeständen liegen ${ }^{21}$. In dem Modell der freien Elektronen ist diese erste Näherung bis jetzt noch nicht durchführbar. Man wird jedoch in Analogie zur MO-Methode erwarten, daß die Berücksichtigung der Elektronenwechselwirkung einen ähnlichen Einfluß auf die Oszillatorstärken haben wird. Allerdings dürfte derselbe hier geringer sein als in der MO-Methode, da ja die beiden Terme schon in nullter Näherung getrennt liegen. Die Berücksichtigung der Elektronenwechselwirkung wird daher z.B. eine geringere Beimischung von $2^{2} \mathrm{~A}_{2}$ zu $1{ }^{2} \mathrm{~A}_{2}$ und umgekehrt verursachen als in der MO-Methode, wo dieselbe nach (27) $50 \%$ beträgt.

Schließlich wollen wir zum Abschluß noch die vorliegenden experimentellen Ergebnisse im $\mathrm{Zu}$ - sammenhang mit unseren theoretischen Berechnungen besprechen. Wir werden uns dabei hauptsächlich auf die Ergebnisse der MO-Methode (Abb. 5) und des Modells der freien Elektronen (Abb. 8) stützen.

In der vorstehenden Arbeit von Schüler und Michel ${ }^{1}$ sind zwei Elektronenspektren diskutiert, die dem Benzylradikal zugeordnet werden. Es ist dies einmal ein Emissions-Spektrum mit dem Ursprung bei etwa $22330 \mathrm{~cm}^{-1}$, das von Schüler und Reinebeck ${ }^{22}$ in der positiven Säule einer Glimmentladung mit einer Mischung von Toluol $\left(\mathrm{C}_{6} \mathrm{H}_{5} \mathrm{CH}_{3}\right)$ und Helium beobachtet und zunächst als V-Spektrum bezeichnet wurde. Das zweite Spektrum ist ein Absorptions-Spektrum mit seinem Ursprung bei etwa $31636 \mathrm{~cm}^{-1}$, das von Por te $^{2}$ bei der Photolyse von Toluol gefunden wurde.

In Abb. 3 der vorstehenden Arbeit ${ }^{1}$ sind diese Spektren auf Grund einer ausführlichen Diskussion der experimentellen Befunde in ein Termschema eingezeichnet worden. Zum besseren Vergleich mit den von uns erhaltenen Resultaten sei diese Figur als Abb. 9 hier nochmals wiedergegeben. Der erste angeregte Zustand $1^{2} \mathrm{~A}_{2}$ in Abb. 5

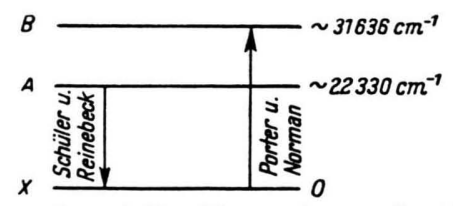

Abb. 9. Experimentelles Termschema des Benzylradikals aus der vorstehenden Arbeit ${ }^{1}$. Die Pfeile geben die beobachteten Übergänge an.

$\left(21300 \mathrm{~cm}^{-1}\right)$ und Abb. $8\left(21800 \mathrm{~cm}^{-1}\right)$ stimmt bezüglich seiner energetischen Lage mit dem ersten Term A von Abb. $9\left(\sim 22330 \mathrm{~cm}^{-1}\right)$ recht gut überein. Der zweite angeregte Term B von Abb. 9 $\left(\sim 31636 \mathrm{~cm}^{-1}\right)$ wäre seiner energetischen Lage nach vielleicht mit den $2^{2} \mathrm{~A}_{2}$-Term von Fig. 5 und 8 zu identifizieren. Diese Zuordnung wird gestützt durch eine Betrachtung der Oszillatorstärken. Die experimentellen Befunde verlangen offenbar, daß $f_{\mathrm{B}} \leftarrow \mathrm{x} \gg f_{\mathrm{A} \leftarrow \mathrm{x}}$ ist, da man sonst den Übergang $\mathrm{A} \leftarrow \mathrm{X}$ auch in Absorption hätte finden müssen. Dieses Verhalten wird nun auch für die obige $\mathrm{Zu}$ ordnung der experimentellen zu den theoretischen Termen von der MO-Methode richtig wiedergegeben, wie die in Abb. 5 angegebenen berechneten Oszillatorstärken zeigen. Mit dem Modell der freien

${ }^{22}$ H. Schüler u. L. Reinebeck, Z. Naturforschg. 6a, 160 [1954]. 
Elektronen ergeben sich bei dieser Zuordnung insofern Schwierigkeiten, als der dazwischenliegende $2^{2} \mathrm{~B}_{2}$-Term im Gegensatz zu den Ergebnissen der MO-Methode eine recht große Oszillatorstärke von $f \approx 0,3$ haben sollte, so da $\beta$ er bei den Absorptionsversuchen ebenfalls hätte gefunden werden müssen. Hierbei ist jedoch zu beachten, daß die hier nicht berücksichtigte Elektronenwechselwirkung die Verteilung der Oszillatorstärken auf $2^{2} \mathrm{~B}_{2}$ und $3^{2} \mathrm{~B}_{2}$ in der oben schon diskutierten Weise so verändert, daß $f_{2^{2} \mathrm{~B}_{2} \leftarrow 1^{2} \mathrm{~B}_{2}} \ll f_{3^{2} \mathrm{~B}_{2} \leftarrow 1^{2} \mathrm{~B}_{2}}$ werden könnte. Der vierte berechnete Term $3{ }^{2} \mathrm{~B}_{2}$ endlich hat zwar nach beiden Methoden eine sehr große Oszillatorstärke, dürfte aber durch die erste Toluolabsorption bei $37500 \mathrm{~cm}^{-1}$ überdeckt werden und daher nur schwierig experimentell zu finden sein.

Die zufriedenstellende Übereinstimmung, die sich bei dem hier durchgeführten Vergleich zwischen den verschiedenen Näherungsverfahren einerseits und zwischen denselben und den spektroskopischen Ergebnissen für das Benzylradikal andererseits ergibt, läßt erwarten, daß solche Berechnungen auch für andere aromatische Radikale erfolgversprechend sind.

\title{
Zur Theorie der magnetischen Sperrschicht in Halbleitern
}

\author{
Von O. Madelung, L. Tewordt und H. Welker
}

Aus dem Forschungslaboratorium der Siemens-Schuckertwerke AG, Erlangen

(Z. Naturforschg. 10a, 476-488 [1955]; eingegangen am 31. März 1955)

Im AnschluB an frühere Arbeiten ${ }^{1}$ wird die Theorie der magnetischen Sperrschicht in Eigenhalbleitern entwickelt und geschlossen dargestellt. Insbesondere wird die Dichteverteilung der Elektron-Loch-Paare unter dem Einfluß der gekreuzten Felder sowie die Strom-Spannungs-Kennlinie und ihre Abhängigkeit von den verschiedenen maßgebenden Größen wie Oberflächenrekombination und Dimensionierung der Probe untersucht. Schließlich werden Fragen wie Einfluß von Lichteinstrahlung, Frequenzverhalten, zeitlicher Auf- und Abbau der Sperrschicht und die Erweiterungsmöglichkeiten der vorliegenden Theorie behandelt.

\section{Qualitatives Bild der magnetischen Sperrschicht}

$\mathrm{W}$ ir fassen noch einmal kurz die wichtigsten qualitativen Züge des hier betrachteten Effektes zusammen:

Wird an einen Eigenhalbleiter ein elektrisches Feld $E_{x}$ und senkrecht dazu ein Magnetfeld $B_{z}$ angelegt, so werden durch die von den Feldern hervorgerufene Lorentz-Kraft Elektronen und Löcher in die gleiche Richtung abgelenkt. Ist in Ablenkungsrichtung infolge freier Oberflächen ein Ladungstransport nicht möglich, so können trotzdem Teilchenströme von Elektronen und Löchern in dieser Richtung fließen; diese müssen aber gleich groß sein, so daß wegen des entgegengesetzten Ladungsvorzeichens der Träger kein elektrischer Strom damit verbunden ist. Sind nun Volumenund Oberflächenrekombination der Halbleiterprobe genügend klein, so werden sich beträchtliche Abweichungen der Elektron-Loch-Paardichte von ihrem Gleichgewichtswert $n_{i}$ einstellen, da sich an

${ }^{1}$ H. Welker, Z. Naturforschg. 6a, 184 [1951]; E. Wei $B$ ha ar u. H. Welker, Z. Naturforschg. 8a, 681 [1953]. der einen Oberfläche Paare stauen und von der anderen Oberfläche her dauernd Paare weggetrieben werden. Dem dabei entstehenden Dichtegradienten wirken zwei Effekte entgegen, die ihn auszuglätten suchen:

a) ein Diffusionsstrom, der in Richtung des negativen Dichtegradienten fließt,

b) die Volumenrekombination von Elektronen und Löchern, die lokal die Dichteerhöhung bzw. -verarmung abzubauen sucht.

Die Randwerte der Dichteverteilung werden durch die Oberflächenrekombinationsgeschwindigkeiten an den beiden freien Oberflächen bestimmt.

Im stationären Fall halten sich also vier Effekte die Waage, die charakterisiert sind durch die GröBen $B_{z} E_{x}$ (Lorentz-Kraft), $D$ (Diffusionskonstante für Elektron-Loch-Paare), $\tau$ (Volumenlebensdauer) und $s$ (Oberflächenrekombinationsgeschwindigkeit). Ferner ist maßgebend für die Dichteverteilung die Dimensionierung der Halbleiter-Probe. Je nach Wahl der charakteristischen Parameter kann sich dabei eine (mittlere) negative Dichteabweichung von $n_{i}$ einstellen (Widerstandsvergröße- 